\title{
EXTINCTION PROFILE OF COMPLETE NON-COMPACT SOLUTIONS TO THE YAMABE FLOW
}

\author{
PANAGIOTA DASKALOPOULOS, JOHN KING, AND NATASA SESUM
}

\begin{abstract}
This work addresses the singularity formation of complete non-compact solutions to the conformally flat Yamabe flow whose conformal factors have cylindrical behavior at infinity. Their singularity profiles happen to be Yamabe solitons, which are self-similar solutions to the fast diffusion equation satisfied by the conformal factor of the evolving metric. The self-similar profile is determined by the second order asymptotics at infinity of the initial data which is matched with that of the corresponding self-similar solution. Solutions may become extinct at the extinction time $T$ of the cylindrical tail or may live longer than $T$. In the first case the singularity profile is described by a Yamabe shrinker that becomes extinct at time $T$. In the second case, the singularity profile is described by a singular Yamabe shrinker slightly before $T$ and by a matching Yamabe expander slightly after $T$.
\end{abstract}

\section{INTRODUCTION}

We consider a complete non-compact metric $g=u^{4 /(N+2)} d x^{2}$ which is conformally equivalent to the standard euclidean metric of $\mathbb{R}^{N}$ and evolves by the Yamabe flow

$$
\frac{\partial g}{\partial t}=-R g
$$

where $R$ denotes the scalar curvature with respect to metric $g$. Our goal is to study the singularity formation of metric $g$ at a singular time $T$, under the assumption that the initial metric $g_{0}$ has cylindrical behavior at infinity.

This flow was introduced by R. Hamilton [18] as an approach to solve the Yamabe problem on manifolds of positive conformal Yamabe invariant. It is the negative $L^{2}$-gradient flow of the total scalar curvature, restricted to a given conformal class. Hamilton [18] showed the existence of the normalized Yamabe flow (which is the re-parametrization of (1.1) to keep the volume fixed) for all time; moreover he established the exponential convergence of the flow to a metric of constant scalar curvature under the assumption that the initial metric has negative scalar curvature.

Since then, there have been a number of works on the convergence of the Yamabe flow on a compact manifold to a metric of constant scalar curvature. Chow [10] showed the convergence of the flow under the conditions that the initial metric is locally conformally flat and of positive Ricci curvature. The convergence of the flow for any locally conformally flat initial metric was shown by Ye [29] (see also a relevant result of Del Pino and Saez [15] for the conformally flat case). 
Schwetlick and Struwe [26] obtained the convergence of the Yamabe flow on a general compact manifold under a suitable Kazdan-Warner type of condition that rules out the formation of bubbles and that is satisfied (via the positive mass Theorem) in dimensions $3 \leq N \leq 5$. The convergence result for any general compact manifold was established by Brendle [3] and [4] (up to a technical assumption, in dimensions $N \geq 6$, on the rate of vanishing of Weyl tensor at the points at which it vanishes): starting with any smooth metric on a compact manifold, the normalized Yamabe flow converges to a metric of constant scalar curvature.

Even though the analogue of Perelman's monotonicity formula is still lacking for the Yamabe flow, one expects that Yamabe soliton solutions model finite time singularities. These are special solutions $g=g_{i j}$ of the Yamabe flow (1.1) for which there exist a potential function $P(x, t)$ so that

$$
(R-\rho) g_{i j}=\nabla_{i} \nabla_{j} P, \quad \rho \in\{1,-1,0\}
$$

where the covariant derivatives on the right hand side are taken with respect to metric $g(\cdot, t)$. Depending on the sign of the constant $\rho$, a Yamabe soliton is called a Yamabe shrinker, a Yamabe expander or a Yamabe steady soliton if $\rho=1,-1$ or 0 respectively. The classification of locally conformally flat Yamabe solitons with positive sectional curvature was recently established in [14] (see also [5] and [8]). It is shown in [14] that such solitons are globally conformally equivalent to $\mathbb{R}^{N}$ and correspond to self-similar solutions of the fast-diffusion equation (1.7) satisfied by the conformal factor. A complete description of those solutions is given in [14]. In [5] the assumption of positive sectional curvature was relaxed to that of nonnegative Ricci curvature.

Our goal in this work is to relate the singularity profile of conformally flat solutions to the Yamabe flow whose conformal factors have cylindrical behavior at infinity with a class of selfsimilar shrinking Yamabe solitons that have matched asymptotic behavior at infinity. One special result in this direction was previously shown in [13] and [2], where the $L^{1}$ stability around the explicit Barenblatt profile was shown.

By observing that the conformal metric $g=u^{4 /(N+2)} d x^{2}$ has scalar curvature

$$
R=-\frac{4(N-1)}{N-2} u^{-1} \Delta u^{\frac{N-2}{N+2}}
$$

it follows that the function $u$ evolves by the fast diffusion equation $u_{t}=\frac{N-1}{m} \Delta u^{m}$, with exponent $m=(N-2) /(N+2)$. Therefore studying the Yamabe flow equation (1.1) in the conformally flat case is equivalent to studying the fast diffusion equation on $\mathbb{R}^{N}$. It is well known [19] that for any exponent $0<m<1$ the Cauchy problem

$$
\begin{cases}u_{t}=\Delta u^{m} & \text { on } \mathbb{R}^{N} \times(0, T) \\ u(\cdot, 0)=u_{0} & \text { on } \mathbb{R}^{N}\end{cases}
$$

with nonnegative and locally integrable initial data $u_{0}$ admits a unique weak solution and that bounded solutions are smooth. We refer the reader to [11] and [9] for extensions of the results in [19] to the case that the initial data is a nonnengative Borel measure $\mu_{0}$ and to 22] for formal 
results that suggest that, within the setting (1.2), many of the phenomena described below are more generally relevant to the range $0<m<\frac{N-2}{N}$ of exponents.

From now on we will fix $m=(N-2) /(N+2)$ and set

$$
n:=1-m=\frac{4}{N+2} \text {. }
$$

We will assume that the initial metric $g_{0}=u_{0}^{4 /(N+2)} d x_{i} d x_{j}$ is complete, non-compact and has cylindrical behavior at infinity, namely

$$
u_{0}(x)=\left(\frac{C^{*} T}{|x|^{2}}\right)^{1 / n}(1+o(1)), \quad \text { as }|x| \rightarrow \infty
$$

with $C^{*}$ given by

$$
C^{*}:=\frac{2(((1-m) N-2)}{n}, \quad n=1-m, \quad m=\frac{N-2}{N+2}
$$

and $T>0$ any positive constant. One observes that the function

$$
\mathcal{C}(x, t)=\left(\frac{C^{*}(T-t)}{|x|^{2}}\right)^{1 / n}
$$

defines a cylindrical solution of (1.2), namely $g(t)=\mathcal{C}^{4 /(N+2)}(\cdot, t) d x^{2}$ represents a shrinking cylindrical metric. Its initial data $\mathcal{C}_{0}:=\mathcal{C}(\cdot, 0)$ satisfies (1.3) and the solution becomes extinct at time $t=T$. This suggests that the cylindrical tail of any solution to (1.2) that satisfies (1.3) becomes extinct at time $T$. Indeed, it will be shown in Proposition 4.1 that if the initial data $u_{0}(x)$ satisfies (1.3) then for the solution $u$ we have

$$
u(x, t)=\left(\frac{C^{*}(T-t)}{|x|^{2}}\right)^{1 / n}(1+o(1)), \quad \text { as }|x| \rightarrow \infty .
$$

We will see in this work that the solution $u$ starting at $u_{0}$ that satisfies (1.3) may or may not become extinct at time $T$, depending on the second order asymptotic behavior, as $|x| \rightarrow \infty$, of the cylindrical tail of the initial data. In either case the metric $g(t)=u^{4 /(N+2)}(\cdot, t) d x^{2}$ will develop a singularity at time $T$. Our goal is to study these singularities. We will show in sections 5 and 6 that rescaled limits of solutions $u$ with initial condition satisfying (1.3) behave near a singularity at time $T$ as self-similar shrinking solutions (Yamabe shrinkers). These are special solutions of the fast-diffusion equation

$$
u_{t}=\Delta u^{\frac{N-2}{N+2}}
$$

of the form

$$
U(x, t)=(T-t)^{\alpha} f(y), \quad y=x(T-t)^{\beta}, \quad \alpha=\frac{1+2 \beta}{n}, \beta>0 .
$$

It follows that the function $f$ satisfies the elliptic equation

$$
\Delta f^{\frac{N-2}{N+2}}+\beta y \cdot \nabla f+\alpha f=0 . \quad \text { on } \mathbb{R}^{N}
$$


It is well known (in [28], Section 3.2.2) that, for any given $\beta>0$ and $\alpha=(1+2 \beta) / n$, equation (1.9) admits an one parameter family $f_{\lambda}, \lambda>0$, of radially symmetric smooth positive solutions that have cylindrical behavior at infinity, namely

$$
f_{\lambda}(y)=\left(\frac{C^{*}}{|y|^{2}}\right)^{1 / n}\left(1+o_{\lambda}(1)\right), \quad \text { as } y \rightarrow \infty .
$$

with $C^{*}$ given by (1.4). We will refer to them as to cigar solitons. The parameter $\lambda$ is just a dilation parameter. Indeed, it follows from the results in [14] that smooth solutions of equation (1.9) are radially symmetric and they are uniquely determined by their value at the origin. In the special case that $\alpha=\beta N$ the solutions are given in the closed form

$$
\mathcal{B}_{\lambda}(y)=\left(\frac{C^{*}}{\lambda^{2}+|y|^{2}}\right)^{1 / n}
$$

and we will refer to them as Barenblatt profiles.

In order to study the singularities of a metric $g=u^{4 /(N+2)} d x^{2}$ evolving by (1.2) and with initial data satisfying (1.3) we need to understand the second order asymptotic behavior at infinity of the self-similar profiles $f_{\lambda}$. We will achieve this in section 3 by linearizing equation (1.9) around the cylindrical solution. It will be more convenient to work in cylindrical coordinates where the cylindrical solution becomes constant. Let $\gamma_{1,2}$ be the solutions to the characteristic equation of the corresponding linearized equation (that is equation (3.7) in section 3). They satisfy

$$
\gamma^{2}+\beta(N-2) \gamma+(N-2)=0
$$

which gives

$$
\gamma_{1,2}=\frac{\beta(N-2) \mp \sqrt{\beta^{2}(N-2)^{2}-4(N-2)}}{2} .
$$

We see that we need to have $\beta \geq 2 / \sqrt{N-2}$ in order for $\gamma_{1,2}$ to be real and the corresponding solution to have non-oscillatory behavior.

Our first result concerns the second order asymptotics of smooth profiles $f$ on $\mathbb{R}^{N}$ which appear to model the singular behavior of some evolving metrics $g=u^{4 /(N+2)} d x^{2}$ that become extinct at a singular time $T$.

Theorem 1.1. Let $m=(N-2) /(N+2), n=1-m, N \geq 3, C^{*}=2((1-m) N-2) / n$, $\beta_{0}:=2 / \sqrt{N-2}$ and $\beta_{1}:=1 /(2 m)$. The following hold:

- Let $N \geq 6$ and $\beta>\beta_{0}$ or $3 \leq N<6$ and $\beta>\beta_{1}$ : For any $B>0$ there exists a unique radially symmetric smooth solution $f_{\beta, B}$ of (1.9) that satisfies

$$
f_{\beta, B}(y)=\left(\frac{C^{*}}{|y|^{2}}\right)^{1 / n}\left(1-B|y|^{-\gamma}+o_{B}\left(|y|^{-\gamma}\right)\right)
$$

with $\gamma=\gamma_{1}$ given by (1.13).

- Let $3 \leq N<6$ and $\beta_{0}<\beta<\beta_{1}$ : For any $B<0$ there exists a unique radially symmetric smooth solution $f_{\beta, B}$ of (1.9) that satisfies (1.14) with $\gamma=\gamma_{1}$ given by (1.13). 
- Let $3 \leq N<6$ and $\beta=\beta_{1}$ : For any $B<0$ there exists a unique radially symmetric smooth solution $f_{\beta, B}$ of (1.9) that satisfies (1.14) with $\gamma=\gamma_{2}=2$ and which is given in closed form by (1.11).

In all of the above cases we will denote by $U_{\beta, B}$ the self-similar solution of equation (1.7). It is given in terms of $f_{\beta, B}$ by (1.8) where $f_{\beta, B}$ solves (1.9).

While the previous theorem provides a complete description of smooth self-similar solutions of equation (1.7) of the form (1.8) with cylindrical behavior at infinity, one may ask whether there exist other radially symmetric self-similar solutions with singular behavior at $r=0$. The answer to this question is indeed affirmative as stated in our next result. We will see in section 6 that such solutions model the behavior of evolving metrics $g=u^{4 /(N+2)} d x_{i} d x_{j}$ that do not become extinct at a singular time $T$, but instead pinch off.

Theorem 1.2. Let $N \geq 3, m=(N-2) /(N+2), n=1-m, C^{*}=2((1-m) N-2) / n$ and $\beta_{1}=1 /(2 m)$. Then for any $\beta>\beta_{1}$ and $B>0$, there exists a unique radially symmetric solution $g_{\beta, B}$ of equation (1.9) that is smooth on $\mathbb{R}^{N} \backslash\{0\}$ and satisfies

$$
g_{\beta, B}(y)=\left(\frac{C^{*}}{|y|^{2}}\right)^{1 / n}\left(1+B|y|^{-\gamma}+o_{B}\left(|y|^{-\gamma}\right)\right), \quad \text { as }|y| \rightarrow \infty
$$

and

$$
g_{\beta, B}(y)=K_{B}|y|^{-\alpha / \beta}(1+o(1)), \quad \text { as }|y| \rightarrow 0
$$

with $K_{B}$ a constant depending on $B$ and $\gamma:=\gamma_{1}$. We will denote by $V_{\beta, B}$ the self-similar solution of equation (1.7) which is given in terms of $g_{\beta, B}$ by (1.8).

One easily concludes, using the behavior of $g_{\beta, B}$ at the origin, that

$$
\lim _{t \rightarrow T^{-}} V_{\beta, B}(x, t)=K_{B}|x|^{-\alpha / \beta} \quad \forall x \neq 0 .
$$

For any $T>0$ and any $K>0$ we will denote by

$$
W_{\beta, K}(x, t)=(t-T)^{\alpha} h_{\beta, K}\left(x(t-T)^{\beta}\right), \quad t>T
$$

the forward self-similar solutions (Yamabe expanders) that satisfy

$$
h_{\beta, K}(y)=K|y|^{-\alpha / \beta}(1+o(1)), \quad \text { as }|y| \rightarrow 0
$$

and

$$
h_{\beta, K}(y)=D_{K}|y|^{-(N+2)}(1+o(1)), \quad \text { as }|y| \rightarrow+\infty
$$

with $D_{K}$ a constant depending on $K$. In [28] Vazquez proves the existence of those solutions starting at $W_{\beta, K}(x, T)=K|x|^{-\alpha / \beta}$. The existence of such solutions and their intermediate asymptotic role was conjectured in [22] on the basis of a phase-plane analysis.

We will see in sections 5 and 6 that the singularity profile of the metric $g=u^{4 /(N+2)} d x_{i} d x_{j}$ evolving by (1.2) at a singular time $T$ is closely related to the self-similar solutions given above. In particular, the smooth self-similar solutions $U_{\beta, B}$ model the singular behavior of some solutions 
$u$ in the case that $u(\cdot, T)$ vanishes identically at time $T$, while the singular solutions $V_{\beta, B}$ and $W_{\beta, K}$ model the singularity of some solutions $u$ in the case that $u(\cdot, T)$ does not vanish identically at the extinction time $T$ of the cylindrical tail.

In describing the asymptotic profile of the solution slightly before time $T$ we will consider the rescaling from the left defined by

$$
\bar{u}(y, \tau):=\left.(T-t)^{-\alpha} u\left(y(T-t)^{-\beta}, t\right)\right|_{t=T\left(1-e^{-\tau}\right)}, \quad(y, \tau) \in \mathbb{R}^{N} \times(0, \infty) .
$$

In describing the asymptotic profile of the solution slightly after time $T$ (if the solution lives for $t \in\left[0, T^{*}\right)$ and $T^{*}>T$ ) we will consider the rescaling from the right defined by

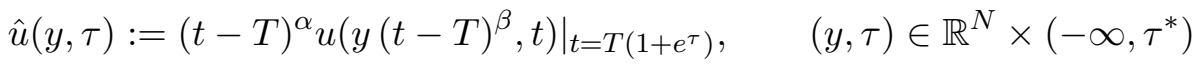

with $\tau^{*}$ such that $T^{*}=T\left(1+e^{\tau^{*}}\right)$. It follows by direct computation that both $\bar{u}$ and $\hat{u}$ satisfy the nonlinear Fokker-Plank type equation

$$
\bar{u}_{\tau}=\Delta \bar{u}^{m}+\beta \operatorname{div}(y \cdot \bar{u})+(\alpha-\beta N) \bar{u} .
$$

Let us begin by discussing the case when the solution with the cylindrical behavior at infinity becomes extinct at the time $T$ when its cylindrical tail disappears. We will assume in this case that either

- $N \geq 3$ and $\beta \geq \beta_{1}$ (or equivalently $N \beta \geq \alpha$ ), or

- $N \geq 6$ and $\beta_{0}<\beta<\beta_{1}$.

The condition $\beta \geq \beta_{0}:=2 / \sqrt{N-2}$ is imposed so that the self similar solution $U_{\beta, B}$ has nonoscillating behavior as $|x| \rightarrow+\infty$. The common feature in both considered cases is that the difference of two self-similar solutions

$$
\left|U_{\beta, B_{1}}-U_{\beta, B_{2}}\right| \notin L^{1}\left(\mathbb{R}^{N}\right), \quad \text { if } B_{1} \neq B_{2} .
$$

The next two theorems generalize the result proved in [13] in the special case when $\beta=\beta_{1}$ (see also in [2] for an improvement of the result in [13] shown independently). Our first result is concerned with the case $\beta \geq \beta_{1}$ in all dimensions $N \geq 3$.

Theorem 1.3. Let $\beta \geq \beta_{1}$ and let $u: \mathbb{R}^{N} \times[0, T) \rightarrow \mathbb{R}$ be a solution to (1.2) with the initial data $u_{0}$ satisfying $0 \leq u_{0} \leq U_{\beta, B_{1}}(\cdot, 0)$, for some $B_{1}>0$. Assume in addition that

$$
u_{0}-U_{\beta, B} \in L^{1}\left(\mathbb{R}^{N}\right)
$$

for some $B>0$. Then, the rescaled solution $\bar{u}$ given by (1.21) converges as $\tau \rightarrow \infty$ uniformly on compact subsets of $\mathbb{R}^{N}$ to the self-similar solution $U_{\beta, B}$. Moreover, we also have convergence in the $L^{1}\left(\mathbb{R}^{N}\right)$ norm. If $\beta>\beta_{1}$ the convergence is exponential.

In the case when $\beta<\beta_{1}$ we will restrict ourselves to $N \geq 6$. Let $\overline{\mathcal{C}}(x)=\left(C^{*} /|x|^{2}\right)^{1 / n}$ with $C^{*}=2((1-m) N-2) / n$ denote the rescaled cylinder which is a singular solution to

$$
\Delta \bar{u}^{m}+\beta \operatorname{div}(x \cdot \nabla \bar{u})=0 .
$$


We define the weighted $L^{1}$-space with weight $\bar{C}^{p_{0}}$ for some $p_{0} \in(0,2 m)$ as

$$
L^{1}\left(\overline{\mathcal{C}}^{p_{0}}, \mathbb{R}^{N}\right):=\left\{f\left|\int_{\mathbb{R}^{N}}\right| f(x) \mid \overline{\mathcal{C}}^{p_{0}}(x) d x<\infty\right\} .
$$

Note that $\overline{\mathcal{C}}^{p_{0}}$ is integrable around the origin for any $p_{0} \in(0,2 m)$. We have the following result.

Theorem 1.4. Let $\beta_{0}<\beta<\beta_{1}$ with $N \geq 6$ and let $u: \mathbb{R}^{N} \times[0, T) \rightarrow \mathbb{R}$ be a solution to (1.2) with the initial data $u_{0}$ satisfying $0 \leq u_{0} \leq U_{\beta, B_{1}}(\cdot, 0)$, for some $B_{1}>0$. Assume in addition that

$$
u_{0}-U_{\beta, B} \in L^{1}\left(\overline{\mathcal{C}}^{p_{0}}, \mathbb{R}^{N}\right)
$$

for some $B>0$, where $p_{0}:=m\left(1-\beta+\sqrt{\beta^{2}-\frac{4}{N-2}}\right)$. Then the rescaled function $\bar{u}$ given by (1.21) converges as $\tau \rightarrow \infty$ uniformly on compact subsets of $\mathbb{R}^{N}$ to the self-similar solution $\bar{U}_{\beta, B}$. Moreover, we also have convergence in the weighted $L^{1}\left(\overline{\mathcal{C}}^{p_{0}}, \mathbb{R}^{N}\right)$.

Remark 1.5. Note that when $\beta<\beta_{1}$ the implication of the $L^{1}$ contraction principle under rescaling (1.21) is inconclusive. The choice of $p_{0}$ as in Theorem 1.4 will allow us to replace the usual $L^{1}$ contraction principle with the weighted $L^{1}$ contraction principle with the weight being $\overline{\mathcal{C}}^{p_{0}}$. Note also that $\left|U_{\beta, B_{1}}-U_{\beta, B_{2}}\right| \notin L^{1}\left(\overline{\mathcal{C}}^{p_{0}}, \mathbb{R}^{N}\right)$ if $B_{1} \neq B_{2}$. This would not be true for any weight $\overline{\mathcal{C}}^{q}$ for $q>p_{0}$.

In section 6 we will discuss the singular behavior of solutions $g=u^{4 /(N+2)} d x_{i} d x_{j}$ to (1.1) with cylindrical behavior at infinity that live past the extinction time $T$ of the cylindrical tail and become compact at time $T$. We will assume that the initial data $u_{0} \in L_{l o c}^{\infty}\left(\mathbb{R}^{N}\right)$ and satisfies

$$
u_{0}(x)-V_{\beta, B}(x) \in L^{1}\left(\mathbb{R}^{N}\right),
$$

where $V_{\beta, B}(x, t)=(T-t)^{\alpha} g_{\beta, B}\left(x(T-t)^{\beta}\right)$ is one of the singular at the origin self similar solutions given by Theorem 1.2 with $\beta>\beta_{1}$ and some $B>0$. In addition we will assume that $u_{0}$ satisfies the asymptotic behavior

$$
u_{0}(x)=\left(\frac{C^{*} T}{|x|^{2}}\right)^{1 / n}\left(1+B|x|^{-\gamma}+o\left(|x|^{-\gamma}\right), \quad \text { as }|x| \rightarrow \infty,\right.
$$

for some $B>0$ and $\gamma:=\gamma_{1}$. We will see that condition (1.27) implies that the solution to (1.2) with initial data $u_{0}$ is strictly positive at the exitinction time $T$ of the cylindrical tail. Denote by $T^{*}>T$ the extinction time of the solution $u$. We have the following result.

Theorem 1.6. Let $\beta \geq \beta_{1}$ and let $u: \mathbb{R}^{N} \times\left[0, T^{*}\right) \rightarrow \mathbb{R}$ be the solution to (1.2) with the initial data $u_{0} \in L_{\text {loc }}^{\infty}\left(\mathbb{R}^{N}\right)$ satisfying (1.27) and (1.28). Then, the following hold

- The solution $u$ is non-zero at time $T$, i.e. $u(\cdot, T)>0$.

- The cylindrical tail becomes extinct at time $T$ according to (1.6) and the rescaled solution $\bar{u}(\eta, \tau)$ given by (1.21) converges as $\tau \rightarrow \infty$ uniformly on compact subsets of $\mathbb{R}^{N}$ to the self-similar profile $g_{\beta, B}(\eta)$ that satisfies (1.15) and (1.16). 
- The rescaled solution $\hat{u}$ given by (1.22) converges as $\tau \rightarrow-\infty$ uniformly on compact subsets of $\mathbb{R}^{N} \backslash\{0\}$ to the self-similar profile $h_{\beta, K}$ that satisfies (1.19) and (1.20) with the same constant $K=K_{B}$ as in (1.16).

- For $t>T$, the solution $u$ satisfies the bound $u(x, t)=O\left(|x|^{-(N+2)}\right)$, as $|x| \rightarrow \infty$.

Remark 1.7. Let $\beta \geq \beta_{1}$ and $\gamma:=\gamma_{1}$. In section 6 we will also see that there exist solutions $g=u^{4 /(N+2)} d x_{i} d x_{j}$ to (1.1) with initial data satisfying $u_{0}-U_{\beta, B} \in L^{1}\left(\mathbb{R}^{N}\right)$ and

$$
u_{0}(x)=\left(\frac{C^{*} T}{|x|^{2}}\right)^{1 / n}\left(1-B|x|^{-\gamma}+o\left(|x|^{-\gamma}\right), \quad \text { as }|x| \rightarrow \infty\right.
$$

with $B>0$ that live longer than the vanishing time $T$ of their cylindrical tail. The rescaling $\bar{u}$ of $u$ given by (1.21) will still converge to $f_{B}$, however, the convergence will only be uniform on $\mathbb{R}^{N} \backslash\{0\}$, reflecting the non-vanishing of the solution at time $T$. This in particular shows that the upper bound $u_{0} \leq U_{\beta, B_{1}}$ in Theorem 1.3 is necessary.

Further Discussion. It would be nice to understand better the singularity formation of the Yamabe flow on complete non-compact manifolds. One of the ultimate goals would be to show that the singularity of such a flow is modeled by one of the gradient Yamabe solitons. For a general statement like that some sort of monotonicity formula would play an important role. By the results in [5, 14] the gradient Yamabe solitons with nonnegative Ricci curvature are well understood and have been shown to be globally conformally equivalent to $\mathbb{R}^{N}$. However, the class of solutions discussed in Theorem 1.6 provides prototypes of singularities that are not globally conformally flat. By the results in [5, 14] the Ricci curvature of those solutions must change sign. A characterization of all gradient Yamabe solitons is then necessary.

The organization of the paper is as follows. In section 2 we discuss the existence of smooth self-similar solutions $U_{\beta, B}$ and singular self-similar solutions $V_{\beta, B}$ and $W_{\beta, K}$. In section 3 we prove Theorem 1.1 and Theorem 1.2. In section 4 we prove Proposition 4.1, which claims the cylindrical tail in a solution persists up to the vanishing time of the cylinder. The proofs of Theorems 1.3 and 1.4 are given in section 5. These theorems discuss the asymptotic profile of solutions that become extinct at the time that their cylindrical tail disappears. In section 6 we discuss solutions that live longer than the time of disappearance of their cylindrical tail and we show the precise singularity profile of those solutions, as stated in Theorem 1.6.

\section{Acknowledgements}

P. Daskalopoulos has been partially supported by NSF grant 0604657. N. Sesum has been partially supported by NSF grants 0905749 and 1056387.

\section{Self-Similar SOLUtions}

Consider self-similar solutions $U(x, t)$ of fast diffusion equation (1.2) in dimensions $N \geq 3$ of the form

$$
U(x, t)=(T-t)^{\alpha} f(y), \quad y=x(T-t)^{\beta}, \quad \alpha=\frac{1+2 \beta}{n}, \quad \beta>0
$$


where $f(y)$ is a radial solution of the elliptic equation (1.9). We recall that we have set $n=1-m$, $m=(N-2) /(N+2)$ and that $N \geq 3$.

In this section we will discuss the existence and geometric properties of three different kinds of self-similar solutions (Yamabe solitions) that will be used in further singularity analysis. In the next section we will discuss their second order asymptotic behavior as $|y| \rightarrow \infty$, which is needed to understand the singular profiles of solutions to (1.2) with cylindrical behavior at infinity. In what follows let $\alpha:=(2 \beta+1) / n$ and $\beta>0$.

(i) We denote by $U_{\beta, B}(x, t)=(T-t)^{\alpha} f_{\beta, B}\left(x(T-t)^{\beta}\right), t \in(-\infty, T)$ and $B>0$ a two parameter family of radially symmetric smooth self-similar solutions satisfying the cylindrical behavior (1.10) at infinity. Their existence for any $\beta>0$ is well known [28].

(ii) We denote by $V_{\beta, B}(x, t)=(T-t)^{\alpha} g_{\beta, B}\left(x(T-t)^{\beta}\right), t \in(-\infty, T)$ and $B>0$ a two parameter family of radially symmetric singular at the origin self-similar solutions with the cylindrical behavior (1.10) at infinity. The behavior of the profile function $g_{\beta, B}$ at the origin is given by (1.16), where $K_{B}$ is a constant depending on $B$. The existence of these solutions will be proved in Proposition 3.5 below.

(iii) We denote by $W_{\beta, K}(x, t)=(t-T)^{\alpha} h_{\beta, K}\left(x(t-T)^{\beta}\right), t \in(T, \infty)$ and $K>0$, a two parameter family of radially symmetric forward self-similar solutions with profile function $h_{\beta, K}$ satisfying (1.19) and (1.20) with $D_{K}$ a constant depending on $K$. In [28] Vazquez proved the existence of these solutions starting with the initial data $W_{\beta, K}(x, T)=K|x|^{-\alpha / \beta}$.

Remark 2.1. In (i) and (ii) above we parametrize the self-similar profiles $f_{\beta, B}$ and $g_{\beta, B}$ by the constant $B$ that appears in second-order asymptotics of the corresponding self-similar solutions (see (1.14) and (1.15)).

2.1. Geometric properties of Yamabe solitons. We summarize below some of the geometric properties of the Yamabe solitons that were introduced above.

- The Yamabe soliton defined by $g=U_{\beta, B}^{\frac{4}{N+2}} d x^{2}$, where $U_{\beta, B}$ is described in (i) above is a complete conformally flat radially symmetric Yamabe shrinker on $\mathbb{R}^{N}$ satisfying equation $(R-1) g_{i j}=\nabla_{i} \nabla_{j} P_{u}$ for a radially symmetric potential function $P_{u}$. This soliton behaves as a cylinder at infinity. In [14] we showed that for $\beta \geq \beta_{1}$ they have positive sectional curvature.

- The Yamabe soliton defined by $g=V_{\beta, B}^{\frac{4}{N+2}} d x^{2}$, where $V_{\beta, B}$ is described in (ii) above is a complete locally conformally flat radially symmetric Yamabe shrinker on $\mathbb{R}^{N} \backslash\{0\}$ satisfying equation $(R-1) g_{i j}=\nabla_{i} \nabla_{j} P_{v}$ for a radially symmetric function $P_{v}$. This soliton also behaves as a cylinder at infinity. It is singular at the origin and therefore by the classification and rigidity result in [7] it has to have somewhere negative Ricci curvature (since it is not flat, not locally isometric to a cylinder, not globally conformally flat and not conformal to a spherical spaceform; the last is true because our soliton is not compact). It is easy to check the completeness of our solution at the origin where 
$V_{\beta, B} \sim|x|^{-\alpha / \beta}$ as $|x| \rightarrow 0$. The completeness follows from

$$
\operatorname{dist}_{g}(x, 0) \geq C \int_{0}^{x}|y|^{-\frac{2 \beta+1}{2 \beta}} d y=+\infty
$$

since $\beta>0$, implying that the distance to the origin is infinity. This soliton has 2 ends.

- The Yamabe soliton defined by $g=W_{\beta, K}^{\frac{4}{N+2}} d x^{2}$, where $W_{\beta, K}$ is described in (iii) above is a complete locally conformally flat radially symmetric Yamabe expander on $\mathbb{R}^{N} \backslash\{0\}$ satisfying equation $(R+1) g_{i j}=\nabla_{i} \nabla_{j} P_{w}$ for a radially symmetric potential function $P_{w}$. This soliton admits the spherical behavior at infinity, which means it is compact on one end. It behaves at the origin like the previously discussed Yamabe shrinker on $\mathbb{R}^{N} \backslash\{0\}$, which means it is complete at the origin (one can also check that the area around the origin is infinite). This soliton metric has only one end and by the same arguments as for the previously discussed Yamabe shrinker has somewhere negative Ricci curvature.

2.2. Scaling and Monotonicity of self-similar solutions. In this section we will use the maximum principle and the scaling properties of equation (1.9) to establish the monotonicity of the self-similar solutions $U_{\beta, B}$ and $V_{\beta, B}$ with respect to the parameter $B$ and with respect to the radial variable $\eta=|y|$.

We begin by noting the relation between the parameter $B$ in the self-similar profiles $f_{\beta, B}$ and $g_{\beta, B}$ (that satisfy (1.14) and (1.15) respectively) and the behavior of those profiles at the origin. To this end, let

$$
U_{\beta, B_{0}}(y, t)=(T-t)^{\alpha} f_{\beta, B_{0}}\left(x(T-t)^{\beta}\right) \quad \text { and } \quad V_{\beta, \bar{B}_{0}}(y, t)=(T-t)^{\alpha} g_{\beta, \bar{B}_{0}}\left(x(T-t)^{\beta}\right)
$$

denote the self-similar solutions with

$$
f_{\beta, B_{0}}(0)=1 \quad \text { and } \quad \lim _{|y| \rightarrow 0}|y|^{\alpha / \beta} g_{\beta, \bar{B}_{0}}(y)=1
$$

respectively. Clearly, $B_{0}=B_{0}(\beta, N)>0$ and $\bar{B}_{0}=\bar{B}_{0}(\beta, N)>0$. Both profiles satisfy (1.14) and (1.15) respectively, which in particular imply that

$$
\lim _{|y| \rightarrow+\infty}|y|^{2 / n} f_{\beta, B_{0}}(y)=\lim _{|y| \rightarrow+\infty}|y|^{2 / n} g_{\beta, \bar{B}_{0}}(y)=C^{*} .
$$

The rescaled solutions of equation (1.9) that preserve (2.1) are given by

$$
f_{\beta, B_{0}}^{\lambda}(y):=\lambda^{2 / n} f_{\beta, B_{0}}(\lambda y) \quad \text { and } \quad g_{\beta, \bar{B}_{0}}^{\lambda}(y):=\lambda^{2 / n} g_{\beta, \bar{B}_{0}}(\lambda y)
$$

and satisfy

$$
f_{\beta, B_{0}}^{\lambda}(0)=\lambda^{2 / n} \quad \text { and } \quad \lim _{|y| \rightarrow 0}|y|^{\alpha / \beta} g_{\beta, \bar{B}_{0}}^{\lambda}(y)=\lambda^{-1 /(n \beta)}
$$

It follows that $f_{\beta, B_{0}}^{\lambda}=f_{\beta, B_{\lambda}}$ and $g_{\beta, \bar{B}_{0}}^{\lambda}=g_{\beta, \bar{B}_{\lambda}}$ where $f_{\beta, B_{\lambda}}$ and $g_{\beta, B_{\lambda}}$ satisfy (1.14) and (1.15) respectively with $B_{\lambda}=B_{0} \lambda^{-\gamma}$ and $\bar{B}_{\lambda}=\bar{B}_{0} \lambda^{-\gamma}$. To simplify the notation in what follows we set $f_{\lambda}(\eta):=f_{\beta, B_{0}}^{\lambda}(y)$ and $g_{\lambda}(\eta):=g_{\beta, \bar{B}_{0}}^{\lambda}(y), \eta=|y|$.

Lemma 2.2. Assume that $N \beta \geq \alpha$ (or equivalently, $\beta \geq \beta_{1}$ ). If $0<\lambda_{1}<\lambda_{2}$, then

$$
f_{\lambda_{1}}<f_{\lambda_{2}} \quad \text { and } \quad g_{\lambda_{1}}>g_{\lambda_{2}} .
$$


Proof. We observe that in the case $N \beta \geq \alpha$ radially symmetric weak solutions of equation (1.9) cannot cross: if they coincide at a point $\eta_{0}=\left|y_{0}\right|$ they must be the same. This follows by the simple observation that uniqueness of weak solutions of equation (1.9) holds on $B_{r_{0}}(0)$ when $N \beta>\alpha$. Assuming that $\lambda_{1}<\lambda_{2}$, it then follows from (2.2) that (2.3) hold.

As a consequence of the previous lemma, we have the following.

Proposition 2.3. Assume that $N \beta \geq \alpha$ (or equivalently, $\beta \geq \beta_{1}$ ). For any $0<B_{1}<B_{2}$ we have

$$
U_{\beta, B_{2}}<U_{\beta, B_{1}}<\mathcal{C}<V_{\beta, B_{1}}<V_{\beta, B_{2}}
$$

with $\mathcal{C}$ denoting the cylindrical solution given by (1.5). In addition, if $\eta=|y|$

$$
\frac{d}{d \eta}\left(\eta^{2 / n} f_{\beta, B}(\eta)\right)>0 \quad \text { and } \quad \frac{d}{d \eta}\left(\eta^{2 / n} g_{\beta, B}(\eta)\right)<0
$$

holds for any $B>0$.

Proof. Similarly to the proof of the previous lemma, any two solutions from the $f_{\beta, B_{i}}$ and $g_{\beta, B_{i}}$, $i=1,2$, of (1.9) cannot cross each other when $N \beta>\alpha$. Hence, for any $0<B_{1}<B_{2}$ the monotonicity $U_{\beta, B_{2}}<U_{\beta, B_{1}}<V_{\beta, B_{1}}<V_{\beta, B_{2}}$ readily follows from the behavior of those solutions at infinity. In addition, the monotonicity of the profiles $f_{\lambda}$ and $g_{\lambda}$, noted in the previous lemma, readily implies that (2.4) holds. This in particular implies that, for any $B_{1}, B_{2}>0$, we have $U_{\beta, B_{1}}<\mathcal{C}<V_{\beta, B_{2}}$ and the proof of the proposition is complete.

\section{PRECise ASYMPtotics OF SELF-Similar SOlUtions}

We will establish in this section the precise asymptotics, up to second order, of the smooth self-similar solutions $U_{\beta, B}$ and the singular self-similar solutions $V_{\beta, B}$, both of which have been discussed in the previous section. More precisely, we will prove Theorems 1.1 and 1.2 .

It will be convenient to work in cylindrical coordinates. Consider for the moment any solution $u$ of fast-diffusion equation (1.7) that is defined on $\mathbb{R}^{N} \times(-\infty, T), T>0$ and vanishes at time $T$. Assuming that $u(r, t)$ is radial, set

$$
v(s, \tau)=(T-t)^{-1 /(p-1)} r^{2 /(p-1)} u^{m}(r, t), \quad r=e^{s}, \quad \tau=-\log (T-t),
$$

where we recall that $p:=1 / m=(N+2) /(N-2)$. Equation (1.2) is equivalent to

$$
\left(v^{p}\right)_{\tau}=v_{s s}+\alpha v^{p}-\bar{\alpha} v, \quad \bar{\alpha}=\frac{(N-2)^{2}}{4}, \quad \alpha=\frac{p}{p-1}=\frac{N+2}{4}
$$

or equivalently

$$
\bar{\alpha}^{-1}\left(v^{p}\right)_{\tau}=\bar{\alpha}^{-1} v_{s s}+\alpha \bar{\alpha}^{-1} v^{p}-v .
$$

Setting $v=\lambda \bar{v}$, we find (after multiplying the above equation by $\lambda^{-1}$ ) that

$$
\bar{\alpha}^{-1} \lambda^{p-1}\left(\bar{v}^{p}\right)_{\tau}=\bar{\alpha}^{-1} v_{s s}+\alpha \lambda^{p-1} \bar{\alpha}^{-1} v^{p}-v .
$$


Choosing $\lambda$ so that $\alpha \lambda^{p-1} \bar{\alpha}^{-1}=1$ we finally conclude the following equation for $\bar{v}$ (which we denote again by $v$ )

$$
\alpha^{-1}\left(v^{p}\right)_{\tau}=\bar{\alpha}^{-1} v_{s s}+v^{p}-v
$$

A self-similar solution $U(r, t)$ of (1.2) given by (1.8) corresponds to a traveling wave solution $V:=v(x-\beta \tau)$ of (3.2). It follows that $v$ satisfies the differential equation

$$
\bar{\alpha}^{-1} v_{s s}+\beta(p-1) v^{p-1} v_{s}+v^{p}-v=0 .
$$

We notice that the cylindrical solution $\mathcal{C}$ of equation (1.2) given by (1.5) now corresponds to the constant solution $v=1$ of equation (3.3). To linearize (3.3) around the constant solution $v=1$, we set $v:=1+w$ and we find that $w$ satisfies the differential equation

$$
\bar{\alpha}^{-1} w_{s s}+\beta(p-1)(1+w)^{p-1} w_{s}+(1+w)^{p}-(1+w)=0
$$

or equivalently, since $(p-1) \bar{\alpha}=N-2$,

$$
w_{s s}+\beta(N-2)(1+w)^{p-1} w_{s}+\frac{N-2}{p-1}\left[(1+w)^{p}-(1+w)\right]=0 .
$$

The linearized operator of (3.4) around $w=0$ is

$$
L_{\beta} w:=w_{s s}+\beta(N-2) w_{s}+(N-2) w=0 .
$$

We may write (3.5) as

$$
w_{s s}+\beta(N-2) w_{s}+(N-2) w=f
$$

with

$$
f:=-(N-2)\left(\beta\left[(1+w)^{p-1}-1\right] w_{s}+\frac{1}{p-1}\left[(1+w)^{p}-1-p w\right]\right) .
$$

Observe that

$$
f=-\frac{(N-2)}{p}\left(\beta \phi^{\prime}(s)+\frac{p}{p-1} \phi(s)\right)
$$

where

$$
\phi(w):=(1+w)^{p}-1-p w=c_{p} w^{2}+O\left(w^{3}\right), \quad \text { as } w \rightarrow 0 .
$$

Since $1+w \geq 0$, we have $w \geq-1$ always. We observe that $\phi(w)$ is a convex function of $w$, since $p>1$, and that its only local minimum on $[-1,+\infty)$ is attained at $w=0$ where $\phi(0)=0$. Hence,

$$
\phi(w) \geq 0 \quad \text { for all } w \in[-1,+\infty) .
$$

We next look for solutions of (3.6) of the form $w(s)=C e^{-\gamma s}$. It follows that $\gamma$ satisfies equation (1.12) and its roots $\gamma_{i}, i=1,2$ are given by (1.13). The roots $\gamma_{i}$ are real (which give non-oscillating solutions $w$ ) iff

$$
\beta^{2}(N-2)^{2}-4(N-2) \geq 0 \quad \text { or } \quad \beta \geq \beta_{0}:=\frac{2}{\sqrt{N-2}}
$$

Notice that

$$
\beta_{1}:=\frac{N+2}{2(N-2)} \geq \beta_{0}:=\frac{2}{\sqrt{N-2}}
$$

for all $N \geq 3$ and that $\beta_{1}=\beta_{0}$ iff $N=6$. Also, if $\beta \geq \beta_{0}$, then $\gamma_{2} \geq \gamma_{1}$ and $\gamma_{2}=\gamma_{1}$ iff $\beta=\beta_{0}$. 
3.1. Second-order asymptotics of the smooth self-similar solutions $U_{\beta, B}$. Our goal in this subsection is to prove Theorem 1.1. Assume from now on that $\beta>\beta_{0}$, so that $\gamma_{2}>\gamma_{1}$. Perform the cylindrical change of coordinates (3.1) for $U_{\beta, B}$ and denote by $w(s)$ as above the perturbation of our solution in cylindrical coordinates from the cylinder $v(s) \equiv 1$ when $s \rightarrow+\infty$. Note that the smoothness of our radial solution $U_{\beta, B}$ at the origin implies $w(s) \sim-1$ as $s \rightarrow-\infty$. We may express the solution $w$ of (3.5) using the variation of parameters formula as

$$
w(s)=-e^{-\gamma_{1} s} \int_{-\infty}^{s} \frac{e^{-\gamma_{2} t}}{W(t)} f(t) d t+e^{-\gamma_{2} s} \int_{-\infty}^{s} \frac{e^{-\gamma_{1} t}}{W(t)} f(t) d t
$$

were $W(t)$ denotes the Wronskian determinant of the solutions $e^{-\gamma_{1} t}, e^{-\gamma_{2} t}$ of the homogeneous equation and is equal to $W(t)=\left(\gamma_{1}-\gamma_{2}\right) e^{-\left(\gamma_{1}+\gamma_{2}\right) t}$. It follows that

$$
w(s)=\frac{1}{\gamma_{2}-\gamma_{1}}\left(e^{-\gamma_{1} s} \int_{-\infty}^{s} e^{\gamma_{1} t} f(t) d t-e^{-\gamma_{2} s} \int_{-\infty}^{s} e^{\gamma_{2} t} f(t) d t\right) .
$$

We conclude that

$$
w(s)=-C_{N}\left(A_{1} e^{-\gamma_{1} s} \int_{-\infty}^{s} e^{\gamma_{1} t} \phi(t) d t-A_{2} e^{-\gamma_{2} s} \int_{-\infty}^{s} e^{\gamma_{2} t} \phi(t) d t\right),
$$

with

Set

$$
A_{i}:=\frac{p}{p-1}-\beta \gamma_{i}, i=1,2, \quad C=C(\beta, N), \quad C_{N}:=\frac{(N-2)}{p\left(\gamma_{2}-\gamma_{1}\right)}>0 .
$$

$$
I_{i}(s):=\int_{-\infty}^{s} e^{\gamma_{i} t} \phi(t) d t \quad \text { and } \quad I_{i}=\int_{-\infty}^{+\infty} e^{\gamma_{i} t} \phi(t) d t \leq+\infty
$$

and recall that (3.9) holds.

Lemma 3.1. The following hold:

- $A_{1}>A_{2}$ for $\gamma_{2}>\gamma_{1}$ and $A_{1}=A_{2}$ iff $\gamma_{1}=\gamma_{2}$ (or equivalently iff $\beta=\beta_{0}$ ).

- For $\beta>\beta_{1}$, we have $A_{2}<0<A_{1}$.

- For $\beta=\beta_{1}$, we have $A_{1}=0, A_{2}<0$ if $N<6$ and $A_{1}>0, A_{2}=0$ if $N>6$ and $A_{1}=A_{2}=0$ if $N=6$ (in this case $\gamma_{1}=\gamma_{2}$ ).

- For $\beta_{0}<\beta<\beta_{1}$ we have: $A_{1}>A_{2}>0$, if $N>6$ and $A_{2}<A_{1}<0$, if $N<6$.

Proof. We have

$$
A_{i}(\beta)=\frac{N-2}{2}\left(\frac{p}{2}-\left(\beta^{2} \mp \beta \sqrt{\beta^{2}-\frac{4}{N-2}}\right)\right), \quad i=1,2
$$

hence by direct calculation

$$
A_{i}^{\prime}(\beta)= \pm \frac{N-2}{2} \frac{\left(\beta \mp \sqrt{\beta^{2}-\frac{4}{N-2}}\right)^{2}}{\sqrt{\beta^{2}-\frac{4}{N-2}}} .
$$

Hence, $A_{1}(\beta)$ is increasing and $A_{2}(\beta)$ is decreasing. Also, $A_{1}\left(\beta_{1}\right)>0$ if $N>6$ and $A_{1}\left(\beta_{1}\right)=0$ if $N \leq 6$. Similarly, $A_{2}\left(\beta_{1}\right)=0$ if $N \geq 6$ and $A_{2}\left(\beta_{1}\right)<0$ if $N<6$. Also, $A_{i}\left(\beta_{0}\right)>0$ if $N>6$ and $A_{i}\left(\beta_{0}\right)=0$ if $N=6$ and $A_{i}\left(\beta_{0}\right)<0$ if $N<6$. Hence: 
- If $\beta>\beta_{1}$, then $A_{1}(\beta)>A_{1}\left(\beta_{1}\right) \geq 0$ and $A_{2}(\beta)<A_{2}\left(\beta_{1}\right) \leq 0$.

- If $N>6, \beta_{0}<\beta<\beta_{1}$, we have $A_{1}(\beta)>A_{1}\left(\beta_{0}\right)>0, A_{2}(\beta)>A_{2}\left(\beta_{1}\right)=0$.

- If $N<6, \beta_{0}<\beta<\beta_{1}$, we have $A_{1}(\beta)<A_{1}\left(\beta_{1}\right)=0$ and $A_{2}(\beta)<A_{2}\left(\beta_{0}\right)<0$.

- If $N=6, \beta_{0}=\beta_{1}$ and $A_{1}=A_{2}=0$, if $\beta=\beta_{1}=\beta_{0}$.

Lemma 3.2. Assume $\beta>\beta_{0}$. We have

$$
|w(s)| \leq C e^{-\gamma_{1} s}, \quad \text { for all } s \geq s_{0}
$$

for some constant $C$ depending on $N$ and $\beta$. Moreover, if $N \leq 6$ and $\beta_{0}<\beta<\beta_{1}$ there exists an $s_{0}$ such that $w(s) \geq 0$ for all $s \geq s_{0}$. In all other cases $w(s)<0$ for all $s \in \mathbb{R}$.

Proof. By (3.10) and (3.9) we have

$$
w(s)=-C_{N}\left(A_{1} e^{-\gamma_{1} s} I_{1}(s)-A_{2} e^{-\gamma_{2} s} I_{2}(s)\right),
$$

as $s \rightarrow+\infty$, where $A_{i}$ are given by (3.11), $C_{N}>0$ and $\lim _{s \rightarrow+\infty} w(s)=0$. The case $A_{1}=0$ happens only when $\beta=\beta_{1}$ and the solution is then explicit (Barenblatt solution). Therefore, we may assume from now on that

$$
A_{1} \neq 0
$$

As $s \rightarrow \infty$,

$$
w(s)=-C_{N}\left(\int_{-\infty}^{s}\left(A_{1} e^{-\gamma_{1}(s-t)}-A_{2} e^{-\gamma_{2}(s-t)}\right) \phi(t) d t\right)
$$

Moreover,

$$
\phi(t) \geq 0, \quad \forall t \quad \text { and } \quad e^{-\gamma_{1}(s-t)} \geq e^{-\gamma_{2}(s-t)}, \quad \forall t \leq s .
$$

Recall that $A_{2}<A_{1}$ always when $\beta>\beta_{0}$. Note that $A_{1}>0$ in all cases except when $N \leq 6$ and $\beta_{0}<\beta \leq \beta_{1}$. Since $A_{2}<A_{1}$, it follows from (3.13) that when $A_{1}>0$ we have $w<0$ for all $s$ and

$$
M_{1} \int_{-\infty}^{s} e^{\gamma_{1} t} \phi(t) d t \leq e^{\gamma_{1} s}|w(s)| \leq M_{2} \int_{-\infty}^{s} e^{\gamma_{1} t} \phi(t) d t
$$

with $M_{1}:=A_{1}-\left|A_{2}\right|>0$ and $M_{2}:=A_{1}+\left|A_{2}\right|<\infty$.

We claim that in the case when $N \leq 6$ and $\beta_{0}<\beta<\beta_{1}$ there exists an $s_{0}$ such that $w(s) \geq 0$, for all $s \geq s_{0}$. In order to see that, we multiply equation (3.7) by $e^{\gamma_{1} s}$ and integrate it over $(-\infty, s]$. After integration by parts, using that $-\gamma_{1}$ is a solution to the characteristic equation for (3.6) and that $\gamma_{1}+\gamma_{2}=\beta(N-2)$, we obtain

$$
w_{s}+\gamma_{2} w=-C_{N} e^{-\gamma_{1} s} A_{1} \int_{-\infty}^{s} \phi(t) e^{\gamma_{1} t} d t-C_{N} \phi
$$

Since $A_{1}<0$ in this case and $C_{N} \phi \leq c w^{2}$ for $s \geq s_{0}$ (by (3.9) ) we conclude

$$
w_{s}+\gamma_{2} w \geq C_{N}\left|A_{1}\right| e^{-\gamma_{1} s} I_{1}(s)-c w^{2}
$$

for $s \geq s_{0}$. The above yields that if ever $w\left(s_{0}\right)=0$ for some $s_{0}$, then $\left.w_{s}\right|_{s=s_{0}} \geq 0$, implying that $w(s) \geq 0$ for all $s \geq s_{0}$. Therefore we have two possibilities, either there exists an $s_{0}$ such that 
$w(s) \geq 0$ for all $s \geq s_{0}$, or $w(s)<0$ for all $s$. In the latter case, using that $\lim _{s \rightarrow \infty} w(s)=0$, we can choose a tiny $\epsilon>0$ so that for $s \geq s_{0}$

$$
w_{s}+\left(\gamma_{2}-\epsilon\right) w \geq 0
$$

implying

$$
\left(w e^{\left(\gamma_{2}-\epsilon\right) s}\right)_{s} \geq 0
$$

Since $\gamma_{2}>\gamma_{1}$ we conclude

$$
|w(s)| \leq C e^{-\gamma_{1} s}, \quad s \geq s_{0} .
$$

This would immediately imply that (3.12) holds. Since $A_{1}<0$ this would mean $w(s)>0$ for $s$ sufficiently large, which contradicts our assumption that $w(s) \leq 0$ for all $s$. We conclude that the first possibility always holds, namely $w(s) \geq 0$ for all $s \geq s_{0}$. Since $A_{2}<A_{1}<0$, it follows from (3.13) that

$$
e^{\gamma_{1} s}|w| \leq C_{N}\left|A_{1}\right| \int_{-\infty}^{s} \phi(t) e^{\gamma_{1} t} d t
$$

We will now show that in all cases (3.12) holds. By (3.14) (holding when $A_{1}>0$ ) and (3.17) (holding when $A_{1}<0$ ) it is sufficient to prove that

$$
I_{1}:=\int_{-\infty}^{+\infty} e^{\gamma_{1} t} \phi(t) d t<+\infty
$$

Indeed, assume that $I_{1}=+\infty$ and choose $s_{0}$ sufficiently large that both (3.9) and

$$
\int_{-\infty}^{s_{0}} e^{\gamma_{1} t} \phi(t) d t \leq \int_{s_{0}}^{s} e^{\gamma_{1} t} \phi(t) d t \quad \text { for } s>>1
$$

hold. By (3.14) and (3.17),

$$
|w(s)| \leq C e^{-\gamma_{1} s} \int_{s_{0}}^{s} e^{\gamma_{1} t} w^{2}(t) d t \quad \text { for } s>>1
$$

for some positive constant $C>0$. We conclude that $J_{s_{0}}(s):=\int_{s_{0}}^{s} e^{\gamma_{1} t} \phi(t) d t$ satisfies

$$
J_{s_{0}}^{\prime}(s) \leq 2 C e^{-\gamma_{1} s} J_{s_{0}}(s)^{2} \quad \text { for } s>>1
$$

from which, after we integrate on $[s,+\infty)$ and use that $J_{s_{0}}:=\lim _{s \rightarrow \infty} J_{s_{0}}(s)=+\infty$, we obtain the lower bound

$$
J_{s_{0}}(s) \geq c e^{\gamma_{1} s}
$$

for some absolute $c>0$. Since for $s>>1$ we either have $w \leq 0$ and $A_{1}>0$ or $w \geq 0$ and $A_{1}<0$, this lower bound and (3.15) imply

$$
\left|w_{s}\right|+\left(\gamma_{2}+\epsilon\right)|w| \geq C_{N} e^{-\gamma_{1} s}\left|A_{1}\right| J_{s_{0}}(s) \geq c>0
$$

yielding a contradiction since $\lim _{s \rightarrow \infty} w(s)=0$.

We will finish by showing that $w(s)<0$ for all $s \in \mathbb{R}$ in the cases stated in the lemma. As shown in (3.11), the constant $C_{N}$ in front of $\phi(s)$ in (3.10) is positive. In the case when $\beta>\beta_{1}$ and $N \geq 3$ we have $A_{2}<0<A_{1}$ and therefore (3.10) implies that $w(s)<0$ for all $s$. In the case 
$\beta_{0} \leq \beta<\beta_{1}$ and $N \geq 6$, since $A_{1}>A_{2}>0$ and $\gamma_{1}<\gamma_{2}$, we have $A_{1} e^{-\gamma_{1}(s-t)}-A_{2} e^{-\gamma_{2}(s-t)}>0$. Equation (3.10) implies again that $w(s)<0$ for all $s$.

Lemma 3.3. If $A_{1} \neq 0$, then

$$
w(s)=-C_{N} A_{1} I_{1} e^{-\gamma_{1} s}(1+o(1))
$$

with $I_{1}:=\int_{-\infty}^{+\infty} e^{\gamma_{1} t} \phi(t) d t$ satisfying $0<I_{1}<\infty$.

Proof. We will use (3.13). We first observe that by (3.9) and (3.12) we have

$$
e^{-\gamma_{2} s} I_{2}(s) \leq e^{-\gamma_{2} s} I_{2}\left(s_{0}\right)+2 c_{p} e^{-\gamma_{2} s} \int_{s_{0}}^{s} e^{\gamma_{2} t} w^{2}(t) d t \leq e^{-\gamma_{2} s} I_{2}\left(s_{0}\right)+C e^{-2 \gamma_{1} s} .
$$

Since $I_{1} \neq 0$ and $\gamma_{2}>\gamma_{1}$, it follows from (3.13) that as $s \rightarrow \infty$, (3.18) holds.

The above discussion leads to the following Proposition.

Proposition 3.4. Let $m=(N-2) /(N+2), N \geq 3, \beta_{0}:=2 / \sqrt{N-2}$ and $\beta_{1}=1 /(2 m)$. We have the following:

- For $N \geq 6$ and $\beta>\beta_{0}$ or $2<N<6$ and $\beta>\beta_{1}$ the solution to (3.5) admits the slow behavior $w(s)=-B e^{-\gamma_{1} s}(1+o(1))$ with $B>0$.

- For $2<N<6$ and $\beta_{0}<\beta<\beta_{1}$ the solution to (3.5) admits the slow behavior $w(s)=$ $B e^{-\gamma_{1} s}(1+o(1))$ with $B>0$.

- For $2<N \leq 6$ and $\beta=\beta_{1}$, the solution to (3.5) admits the fast behavior $w(s)=$ $-B e^{-\gamma_{2} s}(1+o(1))$, with $\gamma_{2}=\gamma_{2}\left(\beta_{1}\right)=2, B>0$.

Proof. In the cases where $A_{1} \neq 0$, the Proposition follows from (3.18). In the cases where $A_{1}=0$, we have $\beta=\beta_{1}$ and the solution is given in closed form (Barenblatt solution) and admits the fast behavior $w(s)=-B e^{-2 s}(1+o(1))$.

Proof of Theorem 1.1. The proof of Theorem 1.1 immediately follows from Proposition 3.4 once we write $v(s)=1+w(s)$ and express everything in polar coordinates on $\mathbb{R}^{N}$.

3.2. Asymptotics of the singular self-similar solutions $V_{\beta, B}$. Our goal in this subsection is to prove Theorem [1.2. Before showing the precise asymptotics of those singular self-similar solutions at infinity we will first briefly comment on their existence. Since this is pretty standard we will omit the details and give the references in which the details can be found.

Lemma 3.5. For every $\beta>\beta_{1}$ and $T>0$, there exists one parameter family of self-similar solutions $V_{\beta, B}(x, t)=(T-t)^{\alpha} g_{\beta, B}\left(x(T-t)^{\beta}\right)$ with profile function $g_{\beta, B}(y)$ satisfying (1.16) with $B>0$ and $K_{B}>0$ (depending on $B$ ) so that

$$
g(y)=\left(\frac{C^{*}}{|y|^{2}}\right)^{1 / n}(1+o(1)), \quad \text { as }|y| \rightarrow+\infty
$$


Proof. We will show the existence of a radial solution $g_{\beta, B}(r), r=|y|$ of equation (1.9) on $\mathbb{R}^{N}$ such that $g_{\beta, B}(r)$ satisfies (1.16) and (3.19). Omit for simplicity the subscripts $\beta, B$ in the proof of this Lemma, but keep in mind that $\beta>0$ has been fixed. Rewrite the equation (1.9) in the following form

$$
\frac{1}{m} r^{1-N}\left(r^{N-1} g(r)^{m-1} g^{\prime}(r)\right)^{\prime}+\beta y g^{\prime}(r)+\alpha g(r)=0 .
$$

As in 28] we introduce the following change of variables

$$
y=e^{s}, \quad X(s):=\frac{r g^{\prime}}{g}, \quad Y(s)=y^{2} g^{1-m} .
$$

Then (3.20) is equivalent to the following autonomous system of ODEs

$$
\begin{aligned}
\dot{X} & =(2-N) X-m X^{2}-m(\alpha+\beta X) Y \\
\dot{Y} & =(2+(1-m) X) Y .
\end{aligned}
$$

Set $\theta:=-\alpha / \beta$. This autonomous system has a local solution with

$$
X(-\infty)=-\theta, \quad Y(s) \sim e^{s(-\theta(1-m)+2)}, \quad \text { as } s \rightarrow-\infty .
$$

These are equivalent to saying that initial data satisfy $g(r) \sim r^{-\theta}$ as $y \rightarrow 0$. Take any self-similar profile $f_{\beta, B}$ of a smooth self-similar solution $U_{\beta, B}$ (as in Theorem 1.1). Then $\lim _{r \rightarrow 0} g(r)>$ $f_{\beta, B}(0)$. We claim this implies

$$
g(r)>f_{\beta, B}(r)
$$

for all $r$ as long as the solution $g$ exists. Indeed, if that were not true, there would exist an $r_{0}$ so that (since our solutions are radially symmetric) $g\left(r_{0}\right)=f_{\beta, B}\left(r_{0}\right)$. Since both, $g$ and $f_{\beta, B}$ are weak solutions of the same elliptic equation (1.9), by the uniqueness of the Dirichlet problem on the ball $B_{r_{0}}(0)$ (which holds since $\beta>\beta_{1}$ ) we would have $g \equiv f_{\beta, B}$ on the same ball. This is impossible since $f_{\beta, B}$ is a smooth solution at the origin unlike the singular solution $g$.

On the other hand the Aronson Benilán inequality ([1]), applied to the ancient solution $V_{\beta, B}$ implies $\Delta V_{\beta, B}^{m} \leq 0$. This means that $\Delta g^{m} \leq 0$, which (if $\left.|y|=r\right)$ is equivalent to $\left(r^{N-1}\left(g^{m}\right)_{r}\right)_{r} \leq$ 0 . After integrating this inequality twice from some fixed $r_{0}>0$ to $r$ we get

$$
g^{m}(r) \leq C\left(1+r_{0}^{2-N}\right) .
$$

Combining the estimates (3.22) and (3.23) yields that our solution $g$ remains strictly positive and bounded for all $r>0$ and therefore it defines the global solution to (3.20).

Almost the same phase-plane analysis as in 28. (see chapter 5) implies that by choosing the right orbit the solution $g$ admits the cylindrical behavior $g(r)=\left(C^{*}|r|^{-2}\right)^{1 / n}(1+o(1))$, as $r \rightarrow+\infty$. To see that we find that the critical points of our system (3.21) are

$$
E:=(0,0), \quad C:=(-(N+2), 0), \quad D:=(-2 /(1-m),(N-2) / m) .
$$

The only difference from the analysis in 28$]$ is that we need to exclude the case that the orbit ends at the critical point $C$. If that were to happen, as in [28] we would get that our solution had the spherical behavior at infinity and therefore was in $L^{1}\left(\mathbb{R}^{N}\right)$. We argue by contradiction that this is not possible. Assume $V_{\beta, B}(x, t)=(T-t)^{\alpha} g_{\beta, B}\left(x(T-t)^{\beta}\right)$ and $g_{\beta, B}$ satisfies (1.16) 
and $g(y) \sim|y|^{-(N+2)}$ as $|y| \rightarrow \infty$. Let $U_{s}(x, t):=(T-t)^{\alpha} f_{s}\left(x(T-t)^{\beta}\right)$ be the spherical solution that becomes extinct at time $T$ as well. By the $L^{1}$ contraction principle we have

$$
\int_{\mathbb{R}^{N}}\left|V_{\beta, B}(x, t)-U_{s}(x, t)\right| d x \leq \int_{\mathbb{R}^{N}}\left|V_{\beta, B}(x, 0)-U_{s}(x, 0)\right| d x \leq C<\infty,
$$

where $C>0$ is a uniform constant. This implies that

$$
\int_{\mathbb{R}^{N}}\left|g_{\beta, B}(y)-f_{s}(y)\right| d y \leq C(T-t)^{\beta N-\alpha} \rightarrow 0,
$$

as $t \rightarrow T$, forcing $g_{\beta, B} \equiv f_{s}$, which is impossible. We have used here our assumption that $\beta>\beta_{1}$ (or equivalently $\beta N>\alpha$ ). Similarly as in 28] we conclude the orbit must end at $D$, hence as $s \rightarrow \infty$ we must have $X \rightarrow-2 / n$, which implies the asymptotic behavior (3.19).

By Lemma 3.5 we already know the existence of the singular self-similar solutions $V_{\beta, B}$ for which their profile $g_{\beta, B}$ satisfies (1.16) and (3.19). To complete the proof of Theorem 1.2 it is therefore enough to show (1.15). For this purpose assume $\beta \geq \beta_{1}$.

Proof of Theorem 1.2. We adopt the same notation as in subsection 3.1. Using the variation of parameters formula we can write $w$ as

$$
w(s)=\frac{1}{\gamma_{2}-\gamma_{1}}\left(e^{-\gamma_{1} s} \int_{s_{0}}^{s} e^{\gamma_{1} t} f(t) d t-e^{-\gamma_{2} s} \int_{s_{0}}^{s} e^{\gamma_{2} t} f(t) d t\right) .
$$

Note that we are not able to integrate from $-\infty$ as in the proof of Theorem 1.1 but instead do so from some finite $s_{0}$, the reason being that the singular behavior of $V_{\beta, B}$ at the origin implies that the above integrals are near $-\infty$. Integration by parts yields

$$
w(s)=C_{N}\left(e^{-\gamma_{1} s}\left(\beta \phi\left(s_{0}\right) e^{\gamma_{1} s_{0}}-A_{1} \int_{s_{0}}^{s} e^{\gamma_{1} t} \phi(t) d t\right)-e^{-\gamma_{2} s}\left(\beta \phi\left(s_{0}\right) e^{\gamma_{2} s_{0}}+A_{2} \int_{s_{0}}^{s} e^{\gamma_{2} t} \phi(t) d t\right)\right) .
$$

Recall that for $\beta \geq \beta_{1}$ we have $A_{2} \leq 0 \leq A_{1}$. By Corollary 2.3 we have $w(s)>0$ for all $s \in(-\infty, \infty)$ in the considered case. This, together with $\phi \geq 0$ (as we showed in the proof of Lemma (3.2), implies

$$
0 \leq w(s) \leq C\left(s_{0}\right) e^{-\gamma_{1} s} \quad \text { for all } s .
$$

As in the proof of Lemma 3.3 we can now argue that, if $I\left(s_{0}\right) \neq 0$ for some $s_{0} \in \mathbb{R}$, then

$$
w(s)=C_{N} I\left(s_{0}\right) e^{-\gamma_{1} s}(1+o(1)),
$$

where $I\left(s_{0}\right):=\beta \phi\left(s_{0}\right) e^{\gamma_{1} s_{0}}-A_{1} \int_{s_{0}}^{\infty} e^{\gamma_{1} t} \phi(t) d t$. Since $w>0$ it follows that $I\left(s_{0}\right) \geq 0$. If $I\left(s_{0}\right)>0$ for some $s_{0}$ we are done. Otherwise, we must have

$$
\beta e^{\gamma_{1} s} \phi(s)=A_{1} \int_{s}^{\infty} e^{\gamma_{1} t} \phi d t, \quad \forall s \in(-\infty,+\infty)
$$

implying that

$$
\phi(s)=C e^{-\left(\gamma_{1}+\frac{A_{1}}{\beta}\right) s} .
$$


On the other hand, $\phi(s) \sim w^{p}(s)$ as $s \rightarrow-\infty$ and, by using the change between radial and cylindrical coordinates and the behavior $g_{\beta, B}(y) \sim|y|^{-\alpha / \beta}$ as $|y| \rightarrow 0$, we obtain $w(s) \sim e^{-\left(\frac{\alpha}{\beta}-\frac{1}{p-1}\right) s}$ as $s \rightarrow-\infty$. This together with (3.25) and the definition of $A_{1}$ would imply that

$$
\left(\frac{\alpha}{\beta}-\frac{1}{p-1}\right) p=\gamma_{1}+\frac{A_{1}}{\beta}=\frac{p}{(p-1) \beta} .
$$

A direct calculation shows that this is equivalent to $b=-4 /(N+6)$, which is impossible since $b$ is always positive. This means that $I\left(s_{0}\right) \neq 0$ for some $s_{0}$ finishing our proof.

\section{Cylindrical Behavior of evolving metrics at infinity}

Recall that the cylindrical self-similar solution to (1.7) is given by (1.5) and becomes extinct at time $T$. Both self similar solutions $U_{\beta, B}$ and $V_{\beta, B}$ whose second-order asymptotics have been discussed in section 3 have cylindrical behavior at infinity and they both become extinct at the time when their cylindrical tails become extinct. These suggest that the cylindrical tail of any solution $u$ that satisfies (1.3) will become extinct at time $T$, as shown in the following proposition.

Proposition 4.1. Let $u$ be a nonnegative weak solution of (1.2) with initial data $u_{0} \in L^{\infty}\left(\mathbb{R}^{N}\right)$ satisfying (1.5) with $C^{*}$ given by (1.4) and $n=1-m$. Then, for every $t \in[0, T)$ we have

$$
u(x, t)=\left(\frac{C^{*}(T-t)}{|x|^{2}}\right)^{\frac{1}{n}}(1+o(1)) .
$$

Before we prove this Proposition we will show that the extinction time of any solution $u$ with initial data $u_{0} \in L_{l o c}^{1}\left(\mathbb{R}^{N}\right)$ satisfying (1.5) is at least $T$.

Lemma 4.2. Let $u$ be a solution of (1.2) as in Proposition 4.1. Then, its extinction time $T^{*}$ satisfies $T^{*} \geq T$.

Proof. It is well known that bounded solutions to (1.2) with $u_{0} \geq 0$ are $C^{\infty}$ smooth and strictly positive up to their extinction time $T^{*}$. Hence, we may assume without loss of generality, that the initial data $u_{0}$ are strictly positive and continuous on $\mathbb{R}^{N}$. Let $\epsilon>0$ be an arbitrarily small positive number. By the asymptotics given by (1.5) and the positivity and continuity of $u_{0}$, it follows that we can choose $k>0$ to imply that

$$
u_{0}(x) \geq\left(\frac{C^{*}(T-\epsilon)}{|x|^{2}+k^{2}}\right)^{1 / n} \quad \forall x \in \mathbb{R}^{N} .
$$

By comparison with the Barenblatt solutions (1.11) we have

$$
u(x, t) \geq\left(\frac{C^{*}(T-\epsilon-t)}{|x|^{2}+k^{2}(T-\epsilon-t)^{-2 \beta_{1}}}\right)^{1 / n} \quad \forall(x, t) \in \mathbb{R}^{N} \times[0, T-\epsilon)
$$

where $\beta_{1}:=(N+2) /(2(N-2))$. This implies that the extinction time

$$
T^{*} \geq T-\epsilon .
$$

Since $\epsilon>0$ is arbitrary, let $\epsilon \rightarrow 0$ above to conclude the proof of the Lemma. 
Proof of Proposition 4.1. Let $\epsilon>0$ be arbitary. By (4.1) we have

$$
|x|^{2 / n} u(x, t) \geq\left(\frac{C^{*}(T-\epsilon-t)|x|^{2}}{|x|^{2}+k^{2}(T-\epsilon-t)^{-2 \beta_{1}}}\right)^{1 / n} .
$$

If we let $|x| \rightarrow \infty$ above we get $\liminf _{|x| \rightarrow \infty}|x|^{2 / n} u(x, t) \geq\left(C^{*}(T-\epsilon-t)\right)^{1 / n}$ and by letting $\epsilon \rightarrow 0$ we obtain

$$
\liminf _{|x| \rightarrow \infty}|x|^{2 / n} u(x, t) \geq\left(C^{*}(T-t)\right)^{1 / n} .
$$

On the other hand, it is easy to check that the function

$$
B_{k^{-}}(x, t):=\left(\frac{C^{*}(T-t)}{|x|^{2}-k^{2}(T-t)^{-2 \beta_{1}}}\right)^{1 / n}
$$

solves the equation (1.7) on $\left\{|x|>k(T-t)^{-\beta_{1}}\right\} \times[0, T)$. Let $\epsilon>0$. By our assumption on $u_{0}$ there exists an $r_{0}$ so that for all $|x| \geq r_{0}$ we have

$$
u_{0}(x) \leq\left(\frac{C^{*}(T+\epsilon)}{|x|^{2}}\right)^{1 / n} \leq\left(\frac{C^{*}(T+\epsilon)}{|x|^{2}-k^{2}(T+\epsilon)^{-2 \beta_{1}}}\right)^{1 / n}
$$

Choose $k$ sufficiently large that $k(T+\epsilon)^{-\beta_{1}} \geq r_{0}$ and set

$$
B_{k^{-}}^{\epsilon}(x, t):=\left(\frac{C^{*}(T+\epsilon-t)}{|x|^{2}-k^{2}(T+\epsilon-t)^{-2 \beta_{1}}}\right)^{1 / n}
$$

which solves equation (1.7) for $(x, t) \in\left\{|x|>k(T+\epsilon-t)^{-\beta_{1}}\right\} \times[0, T)$. The rescaled functions

$$
\tilde{u}(y, t):=u\left((T+\epsilon-t)^{-\beta_{1}} y, t\right) \quad \text { and } \quad \tilde{B}_{k^{-}}^{\epsilon}(y, t):=B_{k^{-}}^{\epsilon}\left((T+\epsilon-t)^{-\beta_{1}} y, t\right)
$$

satisfy the equation

$$
\tilde{u}_{t}=(T+\epsilon-t)^{2 \beta_{1}} \Delta \tilde{u}^{m}+\beta_{1}(T+\epsilon-t)^{-1} y \cdot \nabla \tilde{u}
$$

on $Q_{k}:=\{|y|>k\} \times[0, T)$ and $\tilde{u}(y, 0) \leq \tilde{B}_{k^{-}}^{\epsilon}(y, 0)$ for all $|y|>k$, from (4.3) and the choice $k(T+\epsilon)^{-\beta_{1}} \geq r_{0}$. Note also that for every $t \in[0, T)$ and for every $y_{0}$ with $\left|y_{0}\right|=k$

$$
\lim _{y \rightarrow y_{0}} \tilde{u}(y, t) \leq \lim _{y \rightarrow y_{0}} \tilde{B}_{k^{-}}^{\epsilon}(y, t)
$$

since the right hand side is infinite. The comparison principle applied to (4.4) on $Q_{k}$ yields that $\tilde{u}(y, t) \leq \tilde{B}_{k^{-}}^{\epsilon}(y, t)$ on $Q_{k}$, or equivalently $u(x, t) \leq B_{k^{-}}^{\epsilon}(x, t)$ on $\left\{|x|>k(T+\epsilon-t)^{-\beta_{1}}\right\} \times[0, T)$ implying the bound $\lim \sup _{|x| \rightarrow \infty}|x|^{2 / n} u(x, t) \leq\left(C^{*}(T+\epsilon-t)\right)^{1 / n}$. Letting $\epsilon \rightarrow 0$ yields

$$
\limsup _{|x| \rightarrow \infty}|x|^{2 / n} u(x, t) \leq\left(C^{*}(T-t)\right)^{1 / n},
$$

which together with (4.2) implies the statement of the proposition. 


\section{Extinction PRofile of Solutions to the YAmabe Flow}

We assume in this section that

- either $N \geq 3$ and $\beta \geq \beta_{1}:=1 /(2 m)$, or

- $N \geq 6$ and $\beta<\beta_{1}:=1 /(2 m)$ with $\beta>\beta_{0}:=2 / \sqrt{N-2}$.

Our main goal is to prove Theorems 1.3 and 1.4 .

The assumption $u(x, 0) \leq U_{\beta, B_{1}}(x, 0)$, for some $B_{1}>0$, which is assumed to hold in both Theorems, and the comparison principle imply the upper bound

$$
u(x, t) \leq U_{\beta, B_{1}}(x, t) \quad \text { on } \mathbb{R}^{N} \times(0, T) .
$$

In particular, $u$ vanishes at time $T$. The rescaled function $\bar{u}$ given by (1.21) satisfies the rescaled equation (1.23).

5.1. The case $\beta \geq \beta_{1}$. In this section we will give the proof of Theorem 1.3. We begin by stating the $L^{1}$-contraction property for solutions to (1.2), whose proof can be found in [19, 28].

Lemma 5.1 ( $L^{1}$-contraction [19], 28]). For any two non-negative solutions $u_{1}$ and $u_{2}$ of (1.2) with initial data in $L_{\text {loc }}^{1}\left(\mathbb{R}^{N}\right)$, defined on a time interval $[0, T)$, and any two times $t_{1}$ and $t_{2}$ such that $0 \leq t_{1} \leq t_{2}<T$, we have

$$
\int_{\mathbb{R}^{N}}\left|u_{1}\left(x, t_{2}\right)-u_{2}\left(x, t_{2}\right)\right| d x \leq \int_{\mathbb{R}^{N}}\left|u_{1}\left(x, t_{1}\right)-u_{2}\left(x, t_{1}\right)\right| d x
$$

Observing that

$$
\int_{\mathbb{R}^{N}}|u(x, t)-v(x, t)| d x=e^{(\beta N-\alpha) \tau} \int_{\mathbb{R}^{N}}|\bar{u}(y, \tau)-\bar{v}(y, \tau)| d y,
$$

the contraction property implies the following decay estimate.

Corollary 5.2. Let $u(\cdot, t), v(\cdot, t)$ be two solutions to (1.2) and let $\bar{u}(\cdot, \tau), \bar{v}(\cdot, \tau)$ be their rescalings, respectively. Then

$$
\int_{\mathbb{R}^{N}}|\bar{u}(y, \tau)-\bar{v}(y, \tau)| d y \leq e^{-(\beta N-\alpha) \tau} \int_{\mathbb{R}^{N}}|\bar{u}(y, 0)-\bar{v}(y, 0)| d y
$$

for all $\tau \in(0, \infty)$.

Proof of Theorem 1.3. By (5.1) and (1.8) we have

$$
\bar{u}(y, \tau) \leq f_{\beta, B_{1}}(y) \leq f_{\beta, B_{1}}(0) \quad(y, \tau) \in \mathbb{R}^{N} \times(0, \infty)
$$

since the profile $f_{\beta, B_{1}}$ is decreasing in $|y|$. It follows that for any sequence $\tau_{i} \rightarrow \infty$, the sequence of solutions $\bar{u}_{i}(y, \tau):=\bar{u}\left(y, \tau_{i}+\tau\right)$ is uniformly bounded and hence it is equicontinuous on compact subsets of $\mathbb{R}^{N} \times(-\infty,+\infty)$ by well-known equicontinuity result for solutions to fast diffusion equations (see in [12]). Hence, by the Arzela-Ascoli theorem there exists a subsequence (still denoted by $\left.\tau_{i}\right)$ such that $\bar{u}_{i} \rightarrow \bar{u}_{\infty}$ as $i \rightarrow \infty$, uniformly on compact subsets of $\mathbb{R}^{n} \times(-\infty, \infty)$. 
We will next show that, because of our assumption (1.24), we have $\bar{u}_{\infty} \equiv f_{\beta, B}$, with $f_{\beta, B}$ denoting the profile function of the self-similar solution $U_{\beta, B}$ defined by (1.8). To this end we apply (5.2) to our solution $\bar{u}_{i}(y, \tau)$ and to the rescaled self-similar solution $f_{\beta, B}(y)$ to obtain

$$
\int_{\mathbb{R}^{N}}\left|\bar{u}_{i}(y, \tau)-f_{\beta, B}(y)\right| d y \leq e^{-(\beta N-\alpha)\left(\tau_{i}+\tau\right)} \int_{\mathbb{R}^{N}}\left|\bar{u}_{0}(y)-f_{\beta, B}(y)\right| d y .
$$

Note that because of (1.24), we have $\int_{\mathbb{R}^{N}}\left|\bar{u}_{0}(x)-f_{\beta, B}(x)\right| d x<\infty$. Let $i \rightarrow \infty$ in (5.3) to conclude

$$
\int_{\mathbb{R}^{N}}\left|\bar{u}_{\infty}(y, \tau)-f_{\beta, B}(y)\right| d y \leq \lim _{i \rightarrow \infty} \int_{\mathbb{R}^{N}}\left|\bar{u}_{i}(y, \tau)-f_{\beta, B}(y)\right| d y=0 .
$$

This implies $\bar{u}_{\infty} \equiv f_{\beta, B}$.

We conclude that $\bar{u}(y, \tau)$ converges, as $\tau \rightarrow \infty$, uniformly on compact subsets of $\mathbb{R}^{N}$ and also in the $L^{1}\left(\mathbb{R}^{N}\right)$ norm to the self-similar profile $f_{\beta, B}$. The latter convergence is exponential and the exponential rate of convergence is at least $e^{-(N \beta-\alpha) \tau}$.

5.2. The case $\beta_{0}<\beta<\beta_{1}$ and $N \geq 6$. In this section we will give the proof of Theorem 1.4. Let $\eta \in C_{0}^{\infty}(\mathbb{R})$ be a cut off function such that $\eta(y)=1 / 2$ for $|y|<1 / 2$ and $\eta(y)=0$ for $|y|>1$. Let $\eta_{R}(y):=\eta(y / R), \eta_{\epsilon}:=\eta(y / \epsilon)$ and $\eta_{R, \epsilon}:=\eta_{R}(y)+\eta_{\epsilon}(y)$. Note that $\left|\Delta \eta_{R, \epsilon}\right|+\left|\nabla \eta_{R, \epsilon}\right|^{2} \leq C \epsilon^{-2}$ for $\epsilon / 2 \leq|y| \leq \epsilon$ and $\left|\Delta \eta_{R, \epsilon}\right|+\left|\nabla \eta_{R, \epsilon}\right|^{2} \leq C R^{-2}$ for $R / 2 \leq|y| \leq R$.

We start with the following weighted contraction result. We recall the weighted $L^{1}$ space given by (1.25) with $p_{0} \in(0,2 m)$.

Lemma 5.3. Let $\bar{u}, \bar{v}$ be any solutions to (1.21), with initial data $\bar{u}_{0}, \bar{v}_{0}$ respectively, satisfying $\bar{u}_{0}, \bar{v}_{0} \leq f_{\beta, B_{1}}$. If $\max _{\mathbb{R}^{N}}\left|\bar{u}_{0}-\bar{v}_{0}\right| \neq 0$ then

$$
\left\|\left(\bar{u}-\bar{v}_{0}\right)(\cdot, \tau) \overline{\mathcal{C}}^{p_{0}}\right\|_{L^{1}\left(\mathbb{R}^{N}\right)}<\left\|\left(\bar{u}_{0}-\overline{\mathcal{C}}\right) \overline{\mathcal{C}}^{p_{0}}\right\|_{L^{1}\left(\mathbb{R}^{N}\right)} \quad \forall \tau \geq 0 .
$$

Proof. The condition $\bar{u}_{0}, \bar{v}_{0} \leq f_{\beta, B_{1}}$ implies that $\bar{u}(\cdot, \tau), \bar{v}(\cdot, \tau) \leq f_{\beta, B_{1}}$ for all $\tau \geq 0$, since $f_{\beta, B_{1}}$ is a steady state of the rescaled equation (1.21). Recall also that in the case $N \geq 6$ we have shown in Lemma 3.2 that $w(s)<0$ for all $s \in \mathbb{R}$, which is equivalent to

$$
f_{\beta, B_{1}}(y)<\overline{\mathcal{C}}(y) \quad \forall x \in \mathbb{R}^{N}
$$

where $\overline{\mathcal{C}}(y)=\left(C^{*}|y|^{-2}\right)^{1 / n}$ with $C^{*}$ the constant in (1.4) corresponding to the cylindrical metric.

Set $q:=|\bar{u}-\bar{v}|$. A standard application of Kato's inequality implies that

$$
q_{\tau} \leq \Delta(a q)+\beta \operatorname{div}(y q)+(\alpha-N \beta) q
$$

in the distributional sense, where

$$
a(y, \tau):=\int_{0}^{1} \frac{m d \theta}{(\theta \overline{\mathcal{C}}+(1-\theta) \bar{v})^{n}} .
$$

By the bound $\bar{v} \leq f_{\beta, B_{1}}$ and (5.4) we have

$$
a(y, \tau)>\frac{m}{\bar{C}^{n}}=\frac{m|y|^{2}}{C^{*}} .
$$


Let $\eta_{R, \epsilon}$ be the cut off function introduced above. Equation (5.5) and integration by parts yield

$$
\begin{aligned}
\frac{d}{d \tau} \int_{\mathbb{R}^{N}} q \eta_{R, \epsilon} \overline{\mathcal{C}}^{p_{0}} d y & \leq \int_{\mathbb{R}^{N}}\left[a \Delta \overline{\mathcal{C}}^{p_{0}}-\beta \nabla \overline{\mathcal{C}}^{p_{0}} \cdot y+(\alpha-N \beta) \overline{\mathcal{C}}^{p_{0}}\right] q \eta_{R, \epsilon} d y \\
& +\int_{\mathbb{R}^{N}}\left[\Delta \eta_{R, \epsilon} \overline{\mathcal{C}}^{p_{0}} a+2 a \nabla \eta_{R, \epsilon} \cdot \nabla \overline{\mathcal{C}}^{p_{0}}-\beta y \cdot \nabla \eta_{R, \epsilon} \overline{\mathcal{C}}^{p_{0}}\right] q d y .
\end{aligned}
$$

A direct calculation shows that

$$
\Delta \overline{\mathcal{C}}^{p_{0}}=\frac{1}{4}(N+2)\left(C^{*}\right)^{p_{0} / n} p_{0}\left(N\left(p_{0}-2\right)+2\left(p_{0}+2\right)\right)|x|^{-2-(N+2) p_{0} / 2} .
$$

We see that $\Delta \overline{\mathcal{C}}^{p_{0}}<0$ for $p \in(0,2 m)$. Hence by (5.7)

$$
a \Delta \overline{\mathcal{C}}^{p_{0}}<\frac{N-2}{4 C^{*}} p_{0}\left(N\left(p_{0}-2\right)+2\left(p_{0}+2\right)\right) \overline{\mathcal{C}}^{p_{0}} .
$$

Furthermore,

$$
-\beta \nabla \overline{\mathcal{C}}^{p_{0}} \cdot y+(\alpha-N \beta) \overline{\mathcal{C}}^{p_{0}}=\frac{1}{4} \overline{\mathcal{C}}^{p_{0}}\left(2+N+2 \beta\left(2-N+2 \beta\left(2-N+(2+N) p_{0}\right)\right) .\right.
$$

Estimates (5.8) and (5.9) imply

$$
a \Delta \overline{\mathcal{C}}^{p_{0}}-\beta \nabla \overline{\mathcal{C}}^{p_{0}} \cdot x+(\alpha-N \beta) \overline{\mathcal{C}}^{p_{0}}<K_{N} \overline{\mathcal{C}}^{p_{0}}
$$

with

$$
K_{N}:=\frac{-4-2 \beta(N-2)^{2}+N^{2}+2(-1+\beta)\left(N^{2}-4\right) p_{0}+(N+2)^{2} p_{0}^{2}}{4(N-2)} .
$$

We see that

$$
a \Delta \overline{\mathcal{C}}^{p_{0}}-\beta y \cdot \nabla \overline{\mathcal{C}}^{p_{0}}+(\alpha-N \beta) \overline{\mathcal{C}}^{p_{0}}<0, \quad \text { if } p_{0} \in\left[p_{1}, p_{2}\right]
$$

with $p_{1}:=m\left(1-\beta-\sqrt{\beta^{2}-\frac{4}{N-2}}\right)$ and $p_{2}:=m\left(1-\beta+\sqrt{\beta^{2}-\frac{4}{N-2}}\right)$. We will choose $p_{0}:=$ $p_{2}$. Since $\beta<\beta_{1}$ and $N \geq 6$, it is easy to check that $p_{1}>0$ and $p_{2}<2 m$, so that $p_{0} \in(0,2 m)$. With this choice of $p_{0}$ we conclude from the above discussion that

$$
\begin{aligned}
\frac{d}{d \tau} \int_{\mathbb{R}^{N}} q \eta_{R, \epsilon} \overline{\mathcal{C}}^{p_{0}} d y & \leq \int_{\mathbb{R}^{N}}\left[\Delta \eta_{R, \epsilon} \overline{\mathcal{C}}^{p_{0}} a+2 a \nabla \eta_{R, \epsilon} \cdot \nabla \overline{\mathcal{C}}^{p_{0}}-\beta y \cdot \nabla \eta_{R, \epsilon} \overline{\mathcal{C}}^{p_{0}}\right] q d y \\
& +\int_{\mathbb{R}^{N}}\left[a \Delta \overline{\mathcal{C}}^{p_{0}}-\beta y \cdot \nabla \overline{\mathcal{C}}^{p_{0}}+(\alpha-N \beta) \overline{\mathcal{C}}^{p_{0}}\right] q \eta_{R, \epsilon} d y \\
& =\int_{|x| \leq \epsilon}\left[\Delta \eta_{R, \epsilon} \overline{\mathcal{C}}^{p_{0}} a+2 a \nabla \eta_{R, \epsilon} \cdot \nabla \overline{\mathcal{C}}^{p_{0}}-\beta y \cdot \nabla \eta_{R, \epsilon} \overline{\mathcal{C}}^{p_{0}}\right] q d y \\
& +\int_{R / 2 \leq|x| \leq R}\left[\Delta \eta_{R, \epsilon} \overline{\mathcal{C}}^{p_{0}} a+2 a \nabla \eta_{R, \epsilon} \cdot \nabla \overline{\mathcal{C}}^{p_{0}}-\beta y \cdot \nabla \eta_{R, \epsilon} \overline{\mathcal{C}}^{p_{0}}\right] q d y \\
& +\int_{\mathbb{R}^{N}}\left[a \Delta \overline{\mathcal{C}}^{p_{0}}-\beta y \cdot \nabla \overline{\mathcal{C}}^{p_{0}}+(\alpha-N \beta) \overline{\mathcal{C}}^{p_{0}}\right] q \eta_{R, \epsilon} d y .
\end{aligned}
$$

Observe that since $p_{0}<2 m$

$$
\begin{aligned}
& \int_{|x| \leq \epsilon}\left[a \Delta \eta_{R, \epsilon} \overline{\mathcal{C}}^{p_{0}}\right.\left.+2 a \nabla \eta_{R, \epsilon} \cdot \nabla \overline{\mathcal{C}}^{p_{0}}-\beta y \cdot \nabla \eta_{R, \epsilon} \overline{\mathcal{C}}^{p_{0}}\right] q d y \\
& \leq C \epsilon^{-\left(\frac{n+2}{2} p_{0}+2\right)}\left|B_{\epsilon}(0)\right| \leq C \epsilon^{n-\left(p_{0} \frac{n+2}{2}+2\right)} \rightarrow 0 \text { as } \epsilon \rightarrow 0 .
\end{aligned}
$$


If we let $\epsilon \rightarrow 0$ in (5.11), then since the $\lim _{\epsilon \rightarrow 0} \eta_{R, \epsilon}=\eta_{R}$, where $\eta_{R}$ is a smooth function with compact support in $R / 2 \leq|y| \leq R$, we obtain

$$
\begin{aligned}
\frac{d}{d \tau} \int_{\mathbb{R}^{N}} q \eta_{R} \overline{\mathcal{C}}^{p_{0}} d y & \leq \int_{R / 2 \leq|x| \leq R}\left[\Delta \eta_{R} \overline{\mathcal{C}}^{p_{0}} a+2 a \nabla \eta_{R} \cdot \nabla \overline{\mathcal{C}}^{p_{0}}-\beta y \cdot \nabla \eta_{R} \overline{\mathcal{C}}^{p_{0}}\right] q d y \\
& +\int_{\mathbb{R}^{N}}\left[a \Delta \overline{\mathcal{C}}^{p_{0}}-\beta y \cdot \nabla \overline{\mathcal{C}}^{p_{0}}+(\alpha-N \beta) \overline{\mathcal{C}}^{p_{0}}\right] q \eta_{R} d y .
\end{aligned}
$$

We claim next that if $\bar{u}_{0}-\bar{v}_{0} \in L^{1}\left(\overline{\mathcal{C}}^{p_{0}}, \mathbb{R}^{N}\right)$ then $\bar{u}(\cdot, s)-\bar{v}(\cdot, s) \in L^{1}\left(\overline{\mathcal{C}}^{p_{0}}, \mathbb{R}^{N}\right)$ for $s \in[0, \tau]$ uniformly in $s$. Indeed recalling that we have chosen $p_{0}=p_{2}$ and integrating the previous estimate over $[0, s]$ (with $s \in[0, \tau]$ ) also using (5.10) we get

$$
\begin{aligned}
& \int_{\mathbb{R}^{N}} q(x, s) \eta_{R} d y-\int_{\mathbb{R}^{N}} q(x, 0) \eta_{R} d y \\
& \leq \int_{0}^{s} \int_{R / 2 \leq|y| \leq R}\left[\Delta \eta_{R} \overline{\mathcal{C}}^{p_{0}} a+2 a \nabla \eta_{R} \cdot \nabla \overline{\mathcal{C}}^{p_{0}}-\beta y \cdot \nabla \eta_{R} \overline{\mathcal{C}}^{p_{0}}\right] q d y d \bar{s} \\
& \leq C(\tau) \int_{0}^{\tau} \int_{R / 2 \leq|y| \leq R}|y|^{-\left(\frac{2\left(1+p_{0}\right)}{1-m}+\gamma\right)} d y d \bar{s} \leq C(\tau) .
\end{aligned}
$$

From the uniform integrability of $q(\cdot, s) \in L^{1}\left(\overline{\mathcal{C}}^{p_{0}}, \mathbb{R}^{N}\right)$ on $s \in[0, \tau]$, we conclude that for any fixed $\tau>0$, we have

$$
\lim _{R \rightarrow \infty} \int_{0}^{\tau} \int_{R / 2 \leq|y| \leq R} q(x, s) \overline{\mathcal{C}}^{p_{0}} d y d s=0 .
$$

On the other hand, since $\left|\Delta \eta_{R}\right| \leq C / R^{2},\left|\nabla \eta_{R}\right| \leq C / R, a(y, \tau) \leq C|y|^{2}$ and $\left|\nabla \bar{C}^{p_{0}}\right| \leq C|y| \bar{C}^{p_{0}}$, (5.13) implies

$$
\frac{d}{d \tau} \int_{\mathbb{R}^{N}} q \eta_{R} \bar{C}^{p_{0}} d y \leq \int_{\mathbb{R}^{N}}\left[a \Delta \overline{\mathcal{C}}^{p_{0}}-\beta y \cdot \nabla \overline{\mathcal{C}}^{p_{0}}+(\alpha-N \beta) \overline{\mathcal{C}}^{p_{0}}\right] q \eta_{R} d y+C \int_{R / 2 \leq|y| \leq R} q \bar{C}^{p_{0}} d y .
$$

Integrating the above differential inequality over $[0, \tau]$ and letting $R \rightarrow \infty$, while using (5.15) and (5.10), gives

$$
\int_{\mathbb{R}^{N}}|\bar{u}-\bar{v}| \bar{C}^{p_{0}} d y<\int_{\mathbb{R}^{N}}\left|\bar{u}_{0}-\bar{v}_{0}\right| \bar{C}^{p_{0}} d y
$$

finishing the proof of the lemma.

Proof of Theorem 1.4. Once we have Lemma [5.3, which is the analogue of Lemma 4.1 in [15] we finish the proof of Theorem 1.4 in the same way as the proof of Theorem 1.2 in [15].

\section{Solutions that Live LONGeR}

In Proposition 4.1 we showed how the cylindrical tail shrinks in solutions that start as being asymptotic to a cylinder at infinity. In Theorems 1.3 and 1.4 we dealt with the extinction profile of the class of solutions that become extinct at the time that their cylindrical tail disappears. In this section we give the proof of Theorem 1.6 that describes the precise extinction profile of a class of solutions that live longer than their cylindrical tail. 
Proof of Theorem 1.6. We claim that given our initial conditions there exists a $B_{1}>0$ such that

$$
u_{0} \leq V_{\beta, B_{1}}(\cdot, 0) \quad \text { on } \mathbb{R}^{N} .
$$

To show the claim first note that by our assumption (1.28) there exist $B_{1}>0$ and $r_{0} \geq 1$ sufficiently large so that

$$
u_{0}(x) \leq V_{\beta, B_{1}}(x, 0) \quad \text { for }|x| \geq r_{0} .
$$

Recall that $V_{\beta, B_{1}}(x, t)=(T-t)^{\alpha} g_{B_{1}}\left(x(T-t)^{\beta}\right)$ for $(x, t) \in \mathbb{R}^{N} \times(-\infty, T)$ and the behavior of $g_{B_{1}}$ has been discussed in Theorem 1.2. On the other hand recall that in section 2.2 we defined $g_{B_{\lambda}}(y):=\lambda^{2 / n} g_{B_{0}}(\lambda y)$ where $B_{0}$ is chosen so that the $\lim _{y \rightarrow 0}|y|^{\alpha / \beta} g_{B_{0}}(y)=1$. Recall that $\alpha=(2 \beta+1) / n$. We also have $B_{\lambda}=B_{0} \lambda^{-\gamma}$. Using that $\lim _{\lambda \rightarrow 0}(\lambda|y|)^{\alpha / \beta} g_{B_{0}}(\lambda y)=1$ we have

$$
\lim _{\lambda \rightarrow 0} g_{B_{\lambda}}(y)=|y|^{-\alpha / \beta} \lim _{\lambda \rightarrow 0} \lambda^{2 / n-\alpha / \beta}=|y|^{-\alpha / \beta} \lim _{\lambda \rightarrow 0} \lambda^{-1 /(n \beta)}=+\infty .
$$

This convergence is uniform in $y$ on the set $\left\{|y| \leq r_{0}\right\}$. This means that by choosing $\lambda$ sufficiently small (which corresponds to $B_{\lambda}$ sufficiently large) and increasing the previously chosen $B_{1}$, if necessary, so that $B_{1} \geq B_{\lambda}$ we have

$$
u_{0}(x) \leq V_{\beta, B_{1}}(x) \quad \text { for }|x| \leq r_{0} .
$$

This concludes the proof of the claim.

Let $W_{\beta, K_{1}}$ be the corresponding forward solution defined by (1.18) so that

$$
\lim _{t \rightarrow T^{-}} V_{\beta, B_{1}}(x, t)=\lim _{t \rightarrow T^{+}} W_{\beta, K_{1}}(x, t)=K_{1}|x|^{-\alpha / \beta} .
$$

Notice that the above convergence is uniform on compact subsets of $\mathbb{R}^{N} \backslash\{0\}$ and also in $L_{\text {loc }}^{1}\left(\mathbb{R}^{N}\right)$. Since $u_{0} \leq V_{\beta, B_{1}}(\cdot, 0)$, it follows by the comparison principle that

$$
u(x, t) \leq V_{\beta, B_{1}}(x, t) \quad \text { for }(x, t) \in \mathbb{R}^{N} \times[0, T] .
$$

In particular, $u(x, T) \leq \lim _{t \rightarrow T^{-}} V_{\beta, B_{1}}(x, t)=K_{1}|x|^{-\alpha / \beta}$, so again by the comparison principle we have

$$
u(x, t) \leq W_{\beta, K_{1}}(x, t) \quad \text { for } x \in \mathbb{R}^{N}, t>T .
$$

Note that since $u(\cdot, t), V_{\beta, B}(\cdot, t) \in L_{l o c}^{1}\left(\mathbb{R}^{N}\right)$ for all $t \in[0, T)$, we can apply Corollary 5.2 to show that the rescaled solution $\bar{u}$ as in (1.21) satisfies

$$
\int_{\mathbb{R}^{N}}\left|\bar{u}(y, \tau)-g_{\beta, B}(y)\right| d y \leq e^{-(\beta N-\alpha) \tau} \int_{\mathbb{R}^{N}}\left|\bar{u}_{0}(y)-g_{\beta, B}(y)\right| d y .
$$

Our assumption $u_{0}-V_{\beta, B}(\cdot, 0) \in L^{1}\left(\mathbb{R}^{N}\right)$ implies that $\bar{u}_{0}-g_{\beta, B} \in L^{1}\left(\mathbb{R}^{N}\right)$ and by (6.1) we have

$$
\bar{u}(y, \tau) \leq g_{\beta, B_{1}}(y), \quad \text { for }(y, \tau) \in \mathbb{R}^{N} \times[0, T] .
$$

The same arguments as in the proof of Theorem 1.3 imply that $\bar{u}(\cdot, \tau)$ converges as $\tau \rightarrow \infty$ uniformly on compact sets of $\mathbb{R}^{n} \backslash\{0\}$, and also in the $L^{1}$ norm, to the singular self similar profile $g_{\beta, B}(x)$. Moreover, since $\beta N>\alpha$ the $L^{1}$ convergence is exponential. 
We recall that $\lim _{t \rightarrow T-} V_{\beta, B}(x, t)=K|x|^{-\alpha / \beta}$ for some $K>0$. Let $W_{\beta, K}$ be the corresponding forward solution as in (1.18) so that the $\lim _{t \rightarrow T^{+}} W_{\beta, K}=K|x|^{\alpha / \beta}$. By the $L^{1}$ contraction property applied to $u(\cdot, t)-W_{\beta, K}(\cdot, t)$ we have for $t>T$,

$$
\begin{aligned}
\int_{\mathbb{R}^{N}} \mid u(x, t) & -W_{\beta, K}(x, t)\left|d x \leq \lim _{t \rightarrow T^{+}} \int_{\mathbb{R}^{N}}\right| u(x, t)-W_{\beta, K}(x, t) \mid d x \\
& =\lim _{t \rightarrow T^{-}} \int_{\mathbb{R}^{N}}\left|u(x, t)-V_{\beta, B}(x, t)\right| d x \leq \int_{\mathbb{R}^{N}}\left|u_{0}(x)-V_{\beta, B}(x, 0)\right| d x \leq C .
\end{aligned}
$$

Under the rescaling defined by (1.22) the previous estimate becomes

$$
\int_{\mathbb{R}^{N}}\left|\hat{u}(y, \tau)-h_{\beta, K_{1}}(y)\right| d y \leq C e^{(n \beta-\alpha) \tau}
$$

for $\tau \in\left(-\infty, \tau^{*}\right)$ with $\tau^{*}:=\log \left(T^{*} / T\right)$. Moreover the bound (6.2) becomes

$$
0 \leq \hat{u}(y, \tau) \leq h_{\beta, K_{1}}(y) \quad \text { forall }(y, \tau) \in \mathbb{R}^{N} \times\left(-\infty, \tau^{*}\right)
$$

This together with (6.4) yields the convergence of the rescaled solutions $\hat{u}(\cdot, \tau)$ as $\tau \rightarrow-\infty$ uniformly on compact sets of $\mathbb{R}^{N} \backslash\{0\}$ and exponentially in the $L^{1}$ norm to the singular self-similar profile $h_{\beta, K_{1}}$. This in particular shows that $u(\cdot, T)>0$. Finally, since $g_{\beta, K_{1}}(y)=O\left(|y|^{-(N+2)}\right.$, as $|y| \rightarrow \infty$, the same must hold for $u(\cdot, t)$ for $t>T$ since $W_{\beta, K_{1}}$ dominates $u$.

As a consequence of the proofs of the Theorems 1.3, 1.4 and 1.6 we have the following Corollary that in particular gives other examples of solutions that live longer than the extinction time of their cylindrical tail.

Corollary 6.1. Let $u: \mathbb{R}^{N} \times[0, T) \rightarrow \mathbb{R}$ be a solution to (1.2) with the initial data $u_{0} \in L_{\text {loc }}^{\infty}\left(\mathbb{R}^{N}\right)$ satisfying

$$
u_{0}(x)=\left(\frac{C^{*} T}{|x|^{2}}\right)^{1 / n}\left(1-B|x|^{-\gamma}+o\left(|x|^{-\gamma}\right) \quad \text { as }|x| \rightarrow \infty\right.
$$

where $B>0$ and $\gamma:=\gamma_{1}$.

(i) If $\beta \geq \beta_{1}$ and $N \geq 3$ and $u_{0}-U_{\beta, B}(\cdot, 0) \in L^{1}\left(\mathbb{R}^{N}\right)$ for some $B>0$, then we have the same conclusion as in Theorem 1.3 (just replace the $C^{0}$ convergence on compact subsets of $\mathbb{R}^{N}$ by the uniform $C^{0}$ convergence on compact subsets away from the origin).

(i) If $\beta_{0}<\beta<\beta_{1}$ and $N \geq 6$ and $u_{0}-U_{\beta, B}(\cdot, 0) \in L^{1}\left(\mathbb{R}^{N}, \mathcal{C}^{p_{0}}\right)$ for some $B>0$ and $p_{0}$ as in Theorem 1.4, the same conclusion as in Theorem 1.4 holds (just replace the $C^{0}$ convergence on compact subsets of $\mathbb{R}^{N}$ by the uniform $C^{0}$ convergence on compact subsets away from the origin).

Proof. By the same arguments as in the proof of Theorem 1.6 there exists a $B_{1}>0$ so that $u_{0} \leq V_{\beta, B_{1}}(\cdot, 0)$ on $\mathbb{R}^{N}$. By the comparison principle we have $u(x, t) \leq V_{\beta, B_{1}}(x, t)$ for all $(x, t) \in$ $\mathbb{R}^{N} \times[0, T)$. If we apply the rescaling (1.21) to $u$ this bound reads as $\bar{u}(y, \tau) \leq g_{\beta, B_{1}}(y)$ for all $(y, t) \in \mathbb{R}^{N} \times[0, \infty)$. We can now apply the proofs of Theorems 1.3 and 1.4 to get the convergence statements in (i) and (ii) respectively. The only difference is that, since $g_{\beta, B_{1}}$ is singular at the 
origin, we only have uniform convergence on compact subsets of $\mathbb{R}^{N} \backslash\{0\}$, i.e. away from the origin.

Remark 6.2. One easily checks that $\gamma:=\gamma_{1}<(N+2) / 2$. Consider initial data of the form

$$
u_{0}(x):=U_{\beta, B}(x)+f(a x)
$$

for some $a>0$, where $f \in L^{\infty}\left(\mathbb{R}^{N}\right)$ and $f(x)=o\left(|x|^{-(N+2)}\right.$ as $|x| \rightarrow \infty$. Then $\gamma<(N+2) / 2$ implies that

$$
u_{0}(x)=\left(\frac{C^{*} T}{|x|^{2}}\right)^{2 / n}\left(1-B|x|^{-\gamma}+o\left(|x|^{-\gamma}\right)\right.
$$

where $B>0$. As in [14] choose $a$ sufficiently small that the vanishing time of the solution $u$ to (1.2) starting at $u_{0}$ is $T^{*}>T$ while by Proposition 3.4 the cylindrical tail of $u$ becomes extinct at $T$. We still have $u_{0}(x)-U_{\beta, B}(x) \in L^{1}\left(\mathbb{R}^{N}\right)$ so Corollary 6.1 applies to our solution. The same proof of Theorem 1.4 in [14] yields that for $t>T$

$$
u(x, t) \leq c(t)|x|^{-(N+2)} \quad \text { as }|x| \rightarrow+\infty .
$$

\section{REFERENCES}

[1] Aronson, D., Benilan, P., Regularite des solutions de l'quation des milieux poreux dans RN. (French. English summary), C. R. Acad. Sci. Paris Sr. A-B 288 (1979), no. 2, A103A105.

[2] Blanchet, A., Bonforte, M., Dolbeault, J., Grillo, G., Vaźquez, J.L., Asymptotics of the fast diffusion equation via entropy estimates, Arch. Ration. Mech. Anal. 191 (2009), no. 2, 347 ?385.

[3] Brendle, S., Convergence of the Yamabe flow for arbitrary initial energy, J. Differential Geom. 69 (2005), $217-278$.

[4] Brendle, S., Convergence of the Yamabe flow in dimension 6 and higher, Invent. Math. 170 (2007), 541-576.

[5] Cao, H.-D., Sun, X., Zhang, Y., On the structure of gradient Yamabe solitons; arXiv:1108.6316.

[6] Carrillo, J.A., Di Francesco, M., Toscani, G.,Strict concavity of the 2-Wasserstein distance for the porous medium equation by mass-centering, Proc .Amer. Math.Soc. 135 (2007), 353-363.

[7] Carron, G., Herzlich, M., Conformally flat manifolds with non negative Ricci curvature, Compositio Math. 142 (2006), 798-810.

[8] Catino, G., Mantegazza, C., Mazzieri, L., On the global structure of conformal gradient solitons with nonnegative Ricci tensor; arXiv: arXiv:1109.0243.

[9] Chasseigne, E.,Vazquez, J.L., Theory of extended solutions for fast-diffusion equations in optimal classes of data Radiation from singularities, Arch. Ration. Mech. Anal. 164 (2) (2002), 133-187.

[10] Chow, B., The Yamabe flow on locally conformally flat manifolds with positive Ricci curvature, Comm. Pure Appl. Math. 65 (1992), 1003-1014.

[11] Dahlberg, B. E. J., Kenig, C. E., Nonnegative solutions to fast diffusions; Rev. Mat. Iberoamericana 4 (1) (1988), 11-29.

[12] Daskalopoulos, P., Kenig, C. E., Degenerate diffusions: Initial Value Problems and Local Regularity Theory (with Carlos Kenig), Tracts in Mathematics 1, European Mathematical Society, Zürich (2007).

[13] Daskalopoulos, P., Sesum, N., On the extinction profile of solutions to fast diffusion; J. Reine Angew. Math. 622 (2008), 95-119.

[14] Daskalopoulos, P., Sesum, N., Classification of conformally flat Yamabe solitons; Advances in Mathematics, to appear. 
[15] del Pino, M.; Sáez, M., On the extinction profile for solutions of $u_{t}=\Delta u^{(N-2) /(N+2)}$; Indiana Univ. Math. J. 50 (2001), no. 1, 611-628.

[16] Galaktionov, V.,A., Peletier, L.A, Asymptotic Behaviour near Finite-Time Extinction of the Fast-Diffusion Equation; Arch. Rat. Mech. Anal., 139, (1997) 83-98.

[17] Galaktionov, V.,A., Posaskov, S.,A. On the nonlinear "fast diffusion" equation in $\mathbb{R}^{N}$; Soviet.Math.Dokl. 33 (1986), 412-415.

[18] Hamilton, R., Lectures on geometric flows (1989), unpublished.

[19] Herrero, M.,A., Pierre, M. The Cauchy problem for $u_{t}=\Delta u^{m}$ when $0<m<1$; Transactions of the american mathematical society 291 (1985), 145-158.

[20] Hsu, S.-Y., Asymptotic profile of solutions of a singular diffusion equation as $t \rightarrow \infty$; Nonlinear analysis 48 (2002) 781-790.

[21] Kato, T., Perturbation Theory for Linear Operators; Second Edition, Grundlehren der Mathematischen Wissenschaften 132, Springer-Verlag, Berlin-New York, 1976.

[22] King, J.R., Self-similar behavior for the equation of fast nonlinear diffusion, phil. Trans. R. Soc., London, A 343, (1993), pp 337-375.

[23] King, J.R., Extremely high concentration dopant diffusion in silicon, IMA J. Appl. Math. 40 (1998) $163-181$.

[24] Osher, S. J. and Ralston, J.V., $L^{1}$ stability of travelling waves with applications to convective porous media flow, Comm.Pure Appl.Math. 35 (1982) 737-749.

[25] Peletier, M.A. and Zhang, H., Self-similar solutions of the fast-diffusion equation that do not conserve mass, Diff. Int. Eqns. 8 (1995) 2045-2064.

[26] Schwetlick, H.; Struwe, M. Convergence of the Yamabe flow for "large" energies, J. Reine Angew. Math. 562 (2003), 59-100.

[27] Vazquez,J.L., Asymptotic behaviour for the porous medium equation posed in the whole space, J.Evol.Equ. 3(2003), 67-118.

[28] Vázquez, J. L., Smoothing and decay estimates for nonlinear diffusion equations, Oxford Lecture Series in Mathematics and its Applications 33 Oxford University Press, Oxford, 2006.

[29] Ye, R., Global existence and convergence of Yamabe flow. J. Differential Geom. 39 (1994), no. 1, 35-50.

[30] Zhang, H., On a nonlinear singular diffusion problem: convergence to a travelling wave, Nonlinear analysis TMA 19 (1992), 1111-1120.

P. Daskalopoulos: Department of Mathematics, Columbia University, 2990 Broadway, New York, NY 10027, USA.

E-mail address: pdaskalo@math.columbia.edu

J. King: School of Mathematical Sciences, The University of Nottingham, University Park, NotTingham, NG7 2RD

E-mail address: john.king@nottingham.ac.uk

N. Sesum: Department of Mathematics, Rutgers University, 110 Frelinghuysen road, Piscataway, NJ 08854, USA.

E-mail address: natasas@math.rutgers.edu 